\title{
Coherence for invertible objects and multigraded homotopy rings
}

\author{
DANIEL DUGGER
}

\begin{abstract}
We prove a coherence theorem for invertible objects in a symmetric monoidal category (or equivalently, a coherence theorem for symmetric categorical groups). This is used to deduce associativity, skew-commutativity, and related results for multigraded morphism rings, generalizing the well-known versions for stable homotopy groups.
\end{abstract}

18D10; 55Q05, 55U99

\section{Introduction}

In algebraic topology a classical object of study is the stable homotopy ring $\pi_{*}(S)$, which is $\mathbb{Z}$-graded and graded commutative. For any topological space (or spectrum) $X$ the stable homotopy groups $\pi_{*}(X)$ give a bimodule over $\pi_{*}(S)$. The motivation for the present paper comes from wanting to generalize this basic setup to more sophisticated homotopy theories, where the homotopy rings and modules have a more elaborate grading. Standard examples are the categories of $G$-equivariant spectra and the category of motivic spectra. In these settings it has long been realized that it can be advantageous to use a grading by an index having to do with the invertible objects, rather than a grading by integers (which correspond to integral suspensions/desuspensions of the unit object). This paper deals with some fundamental questions that arise in this general situation.

As we explain below, for general grading schema one must take some care over whether the analog of $\pi_{*}(S)$ is indeed associative, and whether the analogs of $\pi_{*}(X)$ are indeed bimodules. Care is also needed in the treatment of graded commutativity. At an even deeper level than these issues, the ring structure on $\pi_{*}(S)$ is not exactly canonical: different choices in the basic setup can result in different isomorphism classes of rings. We approach these issues by proving a general coherence theorem for invertible objects in a symmetric monoidal category; this is the main result of the paper, and is spread across Theorems 1.6, 1.10, 1.13 and 1.14 below. After establishing the coherence result we deduce the basic facts about $\mathbb{Z}^{n}$-graded homotopy rings as consequences. 


\subsection{Introduction to the problem}

Let $(\mathcal{C}, \otimes)$ be a symmetric monoidal category, and let $S$ denote the unit. Given objects $x_{1}, \ldots, x_{n}$ in $\mathcal{C}$, we write $x_{1} \otimes \cdots \otimes x_{n}$ as an abbreviation for

$$
x_{1} \otimes\left(x_{2} \otimes\left(x_{3} \otimes \cdots\left(x_{n-1} \otimes x_{n}\right)\right)\right) .
$$

We also use $x^{k}$ as an abbreviation for $x \otimes x \otimes \cdots \otimes x$ ( $k$ factors). So note that $x^{2} \otimes y^{3} \otimes z$ is an abbreviation for $(x \otimes x) \otimes((y \otimes(y \otimes y)) \otimes z)$, and that by convention $x^{0}=S$. Finally, for each tuple $a=\left(a_{1}, \ldots, a_{n}\right) \in \mathbb{N}^{n}$, write

$$
\underline{x}^{a}=x_{1}^{a_{1}} \otimes \cdots \otimes x_{n}^{a_{n}} .
$$

An object $X$ of $\mathcal{C}$ is called invertible if there is an object $Y$ and an isomorphism $\eta: S \rightarrow Y \otimes X$. We will say that $(Y, \eta)$ is an inverse for $X$. In this situation there is a unique map $\hat{\eta}: X \otimes Y \rightarrow S$ such that the two evident maps from $(X \otimes Y) \otimes X$ to $X$ are the same, and this $\hat{\eta}$ is an isomorphism (see Proposition 4.11 below). If $a \in \mathbb{Z}$, define

$$
X^{a}= \begin{cases}X^{a} \text { (as already defined) } & \text { if } a \geq 0 \\ Y^{-a} & \text { if } a<0 .\end{cases}
$$

Note that given an invertible object $X$, the isomorphism type of $Y$ is uniquely determined; but given a specific choice of $Y$, the map $\eta$ is not uniquely determined; it can be varied by an arbitrary element of $\operatorname{Aut}(S)$.

Let $X_{1}, \ldots, X_{n}$ be a collection of invertible objects in $\mathcal{C}$, with chosen inverses $\left(Y_{1}, \eta_{1}\right), \ldots,\left(Y_{n}, \eta_{n}\right)$. For $a \in \mathbb{Z}^{n}$, define

$$
\underline{X}^{a}=X_{1}^{a_{1}} \otimes \cdots \otimes X_{n}^{a_{n}} .
$$

Assume now that $\mathcal{C}$ is an additive category and the tensor product is an additive functor in each variable. Let $\pi_{*}(S)$ be the $\mathbb{Z}^{n}$-graded abelian group given by $\pi_{a}(S)=\mathcal{C}\left(\underline{X}^{a}, S\right)$. More generally, if $W$ is a fixed object in $\mathcal{C}$ let $\pi_{*}(W)$ be the $\mathbb{Z}^{n}$-graded abelian group given by $\pi_{a}(W)=\mathcal{C}\left(\underline{X}^{a}, W\right)$. One of the goals of this paper is the following.

\subsection{Proposition (1) $\pi_{*}(S)$ is a $\mathbb{Z}^{n}$-graded ring.}

(2) $\pi_{*}(W)$ is a $\mathbb{Z}^{n}$-graded bimodule over $\pi_{*}(S)$.

(3) There exist elements $\tau_{1}, \ldots, \tau_{n} \in \pi_{(0, \ldots, 0)}(S)$ satisfying $\tau_{i}^{2}=1$ such that for all $f \in \pi_{a}(S)$ and $g \in \pi_{b}(S)$, where $a, b \in \mathbb{Z}^{n}$, one has

$$
f g=g f \cdot\left[\tau_{1}^{\left(a_{1} b_{1}\right)} \cdots \tau_{n}^{\left(a_{n} b_{n}\right)}\right]
$$

In fact, $\tau_{i}$ is the trace of the identity map on $X_{i}$ (see Section 3 for the definition of trace). 
1.3 Remark The groups $\pi_{*}(W)$ depend on the choice of objects $X_{1}, \ldots, X_{n}$, and therefore we should probably write $\pi_{*}^{X}(W)$. We will always regard the sequence $\underline{X}$ as being understood, however. Unfortunately, the ring structure from (1) depends on even more than this: it depends on the choices of $\eta_{1}, \ldots, \eta_{n}$. Given only the sequence $\underline{X}$, the number of isomorphism types of different ring structures is parameterized by the set $\operatorname{Aut}(S)^{n}$; see Proposition 7.2 below.

To see the difficulty in (1), assume that $f: \underline{X}^{a} \rightarrow S$ and $g: \underline{X}^{b} \rightarrow S$ are two maps. Of course we may tensor them together to form $f \otimes g: \underline{X}^{a} \otimes \underline{X}^{b} \rightarrow S \otimes S \cong S$. However, this only yields an element in $\pi_{a+b}(S)$ after choosing an isomorphism $\underline{X}^{a} \otimes \underline{X}^{b} \cong \underline{X}^{a+b}$. The trouble is that there are many such isomorphisms, and we cannot just choose one at random. To ensure that $\pi_{*}(S)$ is associative these isomorphisms must be compatible in the sense that some evident pentagons all commute.

Both (1) and (2) follow from the fact that one can choose such isomorphisms in a compatible way. This is not a particularly hard result, but it does require some care. The skew-commutativity in (3) is more difficult, and when exploring this one quickly realizes the desirability of a general coherence theory for invertible objects. This paper develops such a theory.

Let us say a little more about skew-commutativity. Given an invertible object $X$ and a self-map $f: X \rightarrow X$, there is a well-defined invariant $\operatorname{tr}(f) \in \operatorname{End}(S)$ called the trace (see Section 3). Define $\tau_{X}=\operatorname{tr}\left(\mathrm{id}_{X}\right)$ and call this the basic commuter for the object $X$. One can prove in this generality that $\tau_{X} \in \operatorname{Aut}(S)$ and satisfies $\tau_{X}^{2}=\mathrm{id}_{S}$. In fact $\tau$ gives a homomorphism $\operatorname{Pic}(\mathcal{C}) \rightarrow{ }_{2} \operatorname{Aut}(S)$, where $\operatorname{Pic}(\mathcal{C})$ is the group of isomorphism classes of invertible objects and ${ }_{2} \operatorname{Aut}(S)$ denotes the 2-torsion elements in $\operatorname{Aut}(S)$. This homomorphism is a basic invariant of the symmetric monoidal category, and governs all commutativity issues; see Section 4 for more information.

The motivating examples for $\mathcal{C}$ one may wish to keep in mind throughout the paper are the following.

- The $G$-equivariant stable homotopy category, where $G$ is a finite group (or even a compact Lie group). In this case let $V_{1}, \ldots, V_{n}$ be a collection of finite-dimensional, irreducible, real representations for $G$ that represent every isomorphism type. Let $X_{i}=S^{V_{i}}$ be the suspension spectra of the one-point compactifications.

- The motivic stable homotopy category over some chosen ground ring. Here $X_{1}=S^{1,0}$ and $X_{2}=S^{1,1}$ are the two basic motivic spheres. 
1.4 Remark The product on $\pi_{*}(S)$ defined above might look different from the standard composition product that is used for stable homotopy groups. An easy argument shows that the products are, in fact, the same; see Remark 2.2.

\subsection{Coherence results}

Fix an invertible object $X$ with inverse $(Y, \eta)$. Let $w$ be a tensor word in $X$ and $Y$. As a specific example, let us look at the word $w=(X \otimes(X \otimes Y)) \otimes(Y \otimes X)$. Clearly $w \cong X$, but there are different ways to construct such an isomorphism. We might use the chain

$$
w \cong(X \otimes S) \otimes(Y \otimes X) \cong(X \otimes S) \otimes S \cong X,
$$

where we used $\hat{\eta}$ in the first isomorphism and $\eta$ in the second. Or we might use the chain

$$
w \cong(X \otimes S) \otimes(Y \otimes X) \cong X \otimes(Y \otimes X) \cong(X \otimes Y) \otimes X \cong S \otimes X \cong X,
$$

where we have used $\hat{\eta}$ in both the first isomorphism and in the fourth. Are these two composite isomorphisms the same? Are all composite isomorphisms the same?

The answer to the second question depends on how careful we are. If we allow ourselves to use the twist isomorphism $t: X \otimes X \rightarrow X \otimes X$ then it is not necessarily true that all composite isomorphisms will be the same. However, if we agree not to use the twist then we obtain the following result.

1.6 Theorem (Coherence without twists) Let $w_{1}$ and $w_{2}$ be two tensor words in the formal variables $x$ and $y$. Suppose we are given two "formal composites" $f, g: w_{1} \rightarrow w_{2}$, by which we mean composable sequences of the following kinds of maps:

(i) associativity isomorphisms

(ii) unital isomorphisms $S \otimes W \cong W \cong W \otimes S$

(iii) $\eta$ and $\hat{\eta}$

(iv) maps obtained from the above ones by tensoring with identity maps

(v) inverses of any of the above maps

Then the maps $f(\mathcal{C})$ and $g(\mathcal{C})$, obtained by substituting $X$ and $Y$ for $x$ and $y$ and taking the actual composite in $\mathcal{C}$, are equal. 
1.7 Example The awkwardness in the statement of the above proposition is commonplace in coherence results, because one has to eliminate certain accidental compositions from occurring. For example, suppose the invertible object $X$ happens to be its own inverse: ie, suppose $Y=X$. Then both $\eta$ and $(\hat{\eta})^{-1}$ are maps $S \rightarrow X \otimes X$; however, the theorem does not claim that they are the same map. Indeed, on a formal level $\eta$ is a map $S \rightarrow y x$ and $(\hat{\eta})^{-1}$ is a map $S \rightarrow x y$, and so there is no choice of $w_{2}$ for which we can apply the theorem to these two maps.

1.8 Example To complement the above "nonexample" of the proposition, here is a true application. Consider the words $w_{1}=(x \otimes y) \otimes x$ and $w_{2}=x$. There are two formal compositions $w_{1} \rightarrow w_{2}$ we can construct as follows:

$$
\begin{gathered}
(x \otimes y) \otimes(x \otimes y) \stackrel{\hat{\eta} \otimes \hat{\eta}}{\longrightarrow} S \otimes S \stackrel{\cong}{\longrightarrow} S \\
(x y)(x y) \longrightarrow x(y(x y)) \longrightarrow x((y x) y) \stackrel{1 \otimes \eta^{-1} \otimes 1}{\longrightarrow} x(S y) \longrightarrow x y \stackrel{\hat{\eta}}{\longrightarrow} S
\end{gathered}
$$

Note that we omitted the tensor symbols in the second composite for typographical reasons. The proposition guarantees that the corresponding composites give the same map in any symmetric monoidal category, for any invertible object $X$ and inverse $(Y, \eta)$.

1.9 Remark (Canonical isomorphisms) Let $X$ be an invertible object in $\mathcal{C}$ with inverse $(Y, \eta)$. By a "tensor word" $w$ in $X$ and $Y$ we can mean either a formal expression in the symbols " $X$ " and " $Y$ " or the actual object that results when the expression is evaluated in $\mathcal{C}$. We will usually let the reader deduce the meaning from context, but occasionally we will write $w(\mathcal{C})$ for the latter interpretation, the evaluation of the formal word $w$ inside of $\mathcal{C}$. Formal tensor words are best thought of as functors into $\mathcal{C}$ where the allowable inputs are pairs $(X,(Y, \eta))$.

Consider the following statement: given a tensor word $w$ as above, there is a unique $a \in \mathbb{Z}$ for which $w \cong X^{a}$. This is true for formal tensor words, but not necessarily true for their evaluations in $\mathcal{C}$. For example, if our particular object $X$ is its own inverse ( $Y=X$ ) then we have $X \cong X^{1} \cong X^{-1}$ and so the value of $a$ is not unique. But it is not true that the formal word " $X$ " is isomorphic to the formal word " $X^{-1}$ ".

Keeping this nuance of language in mind, we can apply Theorem 1.6 as follows. Given a formal word $w$ in $X$ and $Y$, there is a unique $a \in \mathbb{Z}$ for which $w \cong X^{a}$ (canonical isomorphism of functors) and moreover the isomorphism can be chosen from the class described in Theorem 1.6, in which case it is canonical. In this paper such canonical isomorphisms will always be denoted $\phi$. The provision of these canonical isomorphisms is one of the main uses of coherence. 
We will need a coherence theorem that is more sophisticated than Theorem 1.6. To state this, imagine that one has formal words $w_{1}, w_{2}, \ldots, w_{n}$ in $x$ and $y$ together with a string of maps

$$
w_{1} \stackrel{f_{1}}{\longrightarrow} w_{2} \stackrel{f_{2}}{\longrightarrow} \cdots \longrightarrow w_{n-1} \stackrel{f_{n-1}}{\longrightarrow} w_{n} .
$$

We assume that each $f_{i}$ is one of the following:

(i) an associativity isomorphism

(ii) one of the unital isomorphisms $S \otimes W \cong W \cong W \otimes S$

(iii) a twist map $t_{x, x}: x \otimes x \rightarrow x \otimes x, t_{x, y}: x \otimes y \rightarrow y \otimes x, t_{y, x}: y \otimes x \rightarrow x \otimes y$, $t_{y, y}: y \otimes y \rightarrow y \otimes y$

(iv) either $\eta$ or $\hat{\eta}$

(v) a map obtained from the above ones by tensoring with identity maps

(vi) an inverse of any of the above maps

Let $(w, f)$ denote the tuple of $w_{i}$ 's and $f_{i}$ 's. Define the parity of $(w, f)$ to be the total number of times $t_{x, x}, t_{y, y}, t_{x, y}$ and $t_{y, x}$ appear; that is, the number of $i$ 's for which one of these maps appears as a tensor factor in $f_{i}$.

1.10 Theorem (Coherence with twists) Let $(w, f)$ and $\left(w^{\prime}, f^{\prime}\right)$ be two strings as above, and let $k$ be the length of the first and $l$ the length of the second. Assume that $w_{1}=w_{1}^{\prime}$ and $w_{k}=w_{l}^{\prime}$. If $(w, f)$ and $\left(w^{\prime}, f^{\prime}\right)$ have the same parity, then the composite of the $f_{i}$ 's is equal to the composite of the $f_{j}^{\prime}$ 's in any symmetric monoidal category, when $x$ and $y$ are replaced with an invertible object $X$ and an inverse $(Y, \eta)$.

1.11 Example Consider the composites

$$
\begin{gathered}
S \stackrel{\eta}{\rightarrow} Y \otimes X \stackrel{t_{Y, X}}{\rightarrow} X \otimes Y \stackrel{\hat{\eta}}{\rightarrow} S, \\
S \stackrel{\phi}{\rightarrow}(Y \otimes Y) \otimes(X \otimes X) \stackrel{\text { id } \otimes t_{X}}{\longrightarrow}(Y \otimes Y) \otimes(X \otimes X) \stackrel{\phi^{-1}}{\longrightarrow} S,
\end{gathered}
$$

where $\phi$ is the canonical isomorphism provided by Theorem 1.6. Then Theorem 1.10 states that these two composites are the same. An attempt to prove this directly will quickly demonstrate the nontriviality of Theorem 1.10.

Now we turn to coherence theorems involving several different invertible objects. Suppose again that $X_{1}, \ldots, X_{n}$ are invertible objects in $\mathcal{C}$. For each $i$, let $\left(X_{i}^{-1}, \eta_{i}\right)$ denote a chosen inverse for $X_{i}$. Let $w$ be a tensor word in $X_{1}, \ldots, X_{n}$ and $X_{1}^{-1}, \ldots, X_{n}^{-1}$. It is clear that $w$ is formally isomorphic to $\underline{X}^{a}$ for a uniquely determined $a \in \mathbb{Z}^{n}$. We want a result which says that different ways of constructing such an isomorphism yield the same result. 
1.12 Remark In our statements of the next two results we dispense with the phrasing about formal compositions and their instances inside of a given symmetric monoidal category. However, this language should be taken as implicit in the statements.

1.13 Theorem (Coherence without self-twists, multiobject case) Let $w$ be a tensor word in the symbols $X_{i}$ and $X_{i}^{-1}, 1 \leq i \leq n$. There is an isomorphism $w \cong \underline{X}^{a}$ constructed as a composite of the following kinds of maps, and moreover this isomorphism is unique. The maps we are allowed to use are:

(i) associativity isomorphisms

(ii) unital isomorphisms

(iii) commutativity isomorphisms $X_{i} \otimes X_{j} \rightarrow X_{j} \otimes X_{i}$ and $X_{i} \otimes X_{j}^{-1} \rightarrow X_{j}^{-1} \otimes X_{i}$ for $i \neq j$

(iv) the maps $\eta_{i}$ and $\hat{\eta}_{i}$

(v) maps obtained from (i)-(iv) by tensoring with identities

(vi) all inverses of maps in (i)-(v)

We also have a more general version involving parity checks. Suppose $w_{1}, w_{2}, \ldots, w_{k}$ are tensor words in the $X_{i}$ 's and $X_{i}^{-1}$ 's, and consider a composite

$$
w_{1} \stackrel{f_{1}}{\longrightarrow} w_{2} \stackrel{f_{2}}{\longrightarrow} \cdots \longrightarrow w_{k-1} \stackrel{f_{k-1}}{\longrightarrow} w_{k} .
$$

We assume that each $f_{i}$ is one of the following:

(i) an associativity isomorphism

(ii) one of the unital isomorphisms $S \otimes W \cong W \cong W \otimes S$

(iii) a twist map $t_{X_{i}, X_{j}}: X_{i} \otimes X_{j} \rightarrow X_{j} \otimes X_{i}, t_{X_{i}, X_{j}^{-1}}: X_{i} \otimes X_{j}^{-1} \rightarrow X_{j}^{-1} \otimes X_{i}$, $t_{X_{i}^{-1}, X_{j}}: X_{i}^{-1} \otimes X_{j} \rightarrow X_{j} \otimes X_{i}^{-1}$ or $t_{X_{i}^{-1}, X_{j}^{-1}}: X_{i}^{-1} \otimes X_{j}^{-1} \rightarrow X_{j}^{-1} \otimes X_{i}^{-1}$, where possibly $i=j$

(iv) one of the $\eta_{i}$ 's or $\hat{\eta}_{i}$ 's

(v) a map obtained from the above ones by tensoring with identity maps

(vi) an inverse of any of the above maps

Define the $i$-parity of the string $(w, f)$ to be the total number of times $t_{X_{i}, X_{i}}, t_{X_{i}, X_{i}^{-1}}$, $t_{X_{i}^{-1}, X_{i}}$, and $t_{X_{i}^{-1}, X_{i}^{-1}}$ appear in the $f_{j}$ 's. We have the following.

1.14 Theorem (Coherence with self-twists, multiobject case) Let $(w, f)$ and $\left(w^{\prime}, f^{\prime}\right)$ be two strings as above, where the length of the first is $k$ and the length of the second is $l$. Assume $w_{1}=w_{1}^{\prime}$ and $w_{k}=w_{l}^{\prime}$. If $(w, f)$ and $\left(w^{\prime}, f^{\prime}\right)$ have the same $i$-parity for all $1 \leq i \leq n$, then the composites of the two strings are the same map. 


\subsection{Applications}

The first application answers the question raised at the beginning of the paper. If $f \in \pi_{a}(S)$ and $g \in \pi_{b}(S)$, then form the tensor product $f \otimes g: \underline{X}^{a} \otimes \underline{X}^{b} \rightarrow$ $S \otimes S \cong S$. Theorem 1.13 supplies a canonical isomorphism $\underline{X}^{a} \otimes \underline{X}^{b} \rightarrow \underline{X}^{a+b}$, and using this we obtain an element $f \cdot g \in \pi_{a+b}(S)$. Similarly, one obtains maps $\pi_{a}(S) \otimes \pi_{b}(W) \rightarrow \pi_{a+b}(W)$ and so forth. Coherence guarantees that these pairings all have the desired associativity (see Section 2 for details).

Given a map $f: \underline{X}^{a} \rightarrow \underline{X}^{b}$ there are two evident ways to recover an element of $\pi_{a-b}(S)$. We can tensor on the left with $\underline{X}^{-b}$ and then use the canonical isomorphisms from Theorem 1.13, or we can tensor on the right and use canonical isomorphisms. We call the associated elements $[f]_{r}$ and $[f]_{l}$, respectively. Another application of coherence is to relate these two elements:

$$
[f]_{r}=[f]_{l} \cdot \prod \tau_{i}^{b_{i}\left(a_{i}-b_{i}\right)},
$$

where the $\tau_{i}$ 's are the basic commuters of the $X_{i}$ 's. This and many related formulas are developed in Section 6.

Let us very briefly indicate the idea behind skew-commutativity. If $f: \underline{X}^{a} \rightarrow S$ and $g: \underline{X}^{b} \rightarrow S$ then we may form the diagram:

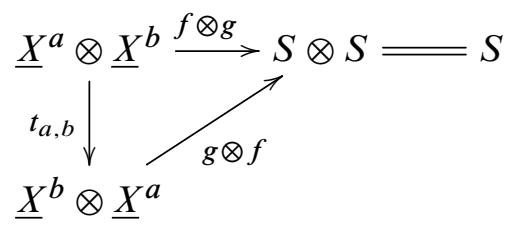

A little work gives that inside $\pi_{*}(S)$ we have $f \cdot g=g \cdot f \cdot\left[t_{a, b}\right]_{r}$ (note that by (1.16) one has $\left.\left[t_{a, b}\right]_{r}=\left[t_{a, b}\right]_{l}\right)$. The content of Proposition 1.2(3) is the identification of $\left[t_{a, b}\right]_{r}$ as a product of basic commuters; this is a direct consequence of Theorem 1.14, which says that the associated composite $S \rightarrow S$ is determined purely by the parities involved; see Section 6 for complete details.

\subsection{The stable motivic homotopy ring}

We close this long introduction with a very specific example. In the stable motivic homotopy category (over a chosen ground field) there are two basic spheres denoted $S^{1,0}$ and $S^{1,1}$. These are invertible objects. More generally one sets $S^{p, q}=\left(S^{1,0}\right)^{\wedge(p-q)} \wedge\left(S^{1,1}\right)^{\wedge(q)}$ for any $p, q \in \mathbb{Z}$. The bigraded stable homotopy ring $\pi_{*, *}(S)$ is an instance of the general situation considered in this paper, although unfortunately the bigrading is different from the generic bigrading we adopted for 
Proposition 1.2: the motivic group $\pi_{a, b}(S)$ corresponds to what we have been calling $\pi_{(a-b, b)}(S)$.

The basic commuter for $S^{1,0}$ is the element $-1 \in \pi_{0,0}(S)$. The basic commuter for $S^{1,1}$ is represented by the twist map $S^{1,1} \wedge S^{1,1} \rightarrow S^{1,1} \wedge S^{1,1}$; in motivic homotopy theory it is usually denoted $\epsilon \in \pi_{0,0}(S)$. The skew-commutativity result for the motivic stable homotopy ring is the following, obtained as a direct corollary of Proposition 1.2.

1.18 Proposition For $f \in \pi_{a, b}(S)$ and $g \in \pi_{c, d}(S)$ one has

$$
f g=g f \cdot(-1)^{(a-b)(c-d)} \cdot \epsilon^{b d} .
$$

Now assume that the ground field is $\mathbb{C}$, so that there is a realization map $\psi$ from the stable motivic homotopy category to the classical stable homotopy category of topological spaces. This induces a collection of group homomorphisms $\psi_{p, q}: \pi_{p, q}(S) \rightarrow \pi_{p}(S)$. One's first guess might be that these maps assemble into a ring homomorphism $\psi: \pi_{*, *}(S) \rightarrow \pi_{*}(S)$, but this is not quite right. Instead there is the following identity.

1.19 Proposition For $f \in \pi_{a, b}(S)$ and $g \in \pi_{c, d}(S)$ one has

$$
\psi(f g)=\psi(f) \cdot \psi(g) \cdot(-1)^{b(c-d)} .
$$

This result was one of the motivations for the work in this paper; we include the proof as a brief appendix.

1.20 Remark It is satisfying to check that Propositions 1.18 and 1.19 are (taken together) compatible with the graded commutativity of the classical stable homotopy ring. This uses that $\psi(\epsilon)=-1$.

\subsection{Generalizations}

Let $\mathrm{Pic}(\mathrm{C})$ be the group of isomorphism classes of invertible objects in $\mathcal{C}$. Let $A$ be an abelian group and let $h: A \rightarrow \operatorname{Pic}(\mathcal{C})$ be a homomorphism (the case $A=\operatorname{Pic}(\mathcal{C})$ is the main one of interest, but it is useful to work in slightly greater generality). The question we pose is whether $\pi(S)$ can be regarded as an $A$-graded ring. We can certainly choose, for each $a \in A$, an object $X_{a}$ in the isomorphism class $h(a)$. We can then define an $A$-graded abelian group by

$$
\pi_{a}^{A}(S)=\mathcal{C}\left(X_{a}, S\right) .
$$


To give a pairing on this graded group one should start by choosing isomorphisms $\sigma_{a, b}: X_{a+b} \rightarrow X_{a} \otimes X_{b}$, and for the pairing to be associative these isomorphisms must satisfy a certain compatibility condition (a unital condition should also be imposed). Our coherence results show that when $A$ is finitely-generated and free this can be accomplished, although it is important to realize that the method for doing so is not quite canonical, depending both on a choice of basis for $A$ and a choice of the $\eta_{i}$ maps we encountered earlier. What about other values of $A$ ? We will show that

(1) for any abelian group $A$, the isomorphisms $\sigma_{a, b}$ can be chosen so that $\pi_{*}^{A}(S)$ is an associative and unital ring;

(2) however, the choices involved in (1) are not canonical and the different isomorphism classes of rings one can obtain are in bijective correspondence with the elements of the group cohomology $H^{2}(A ; \operatorname{Aut}(S))$.

In homotopy theory one often hears the slogan "one should grade things by the invertible objects"; point (2) above suggests that this is a little more dicey than one might wish. These results are in Section 7.

The methods of Section 7 are obstruction-theoretic, and it is interesting that these seem to be very different than the approach via coherence theorems used earlier in the paper for the case where $A$ is free. The following diagram summarizes the situation:

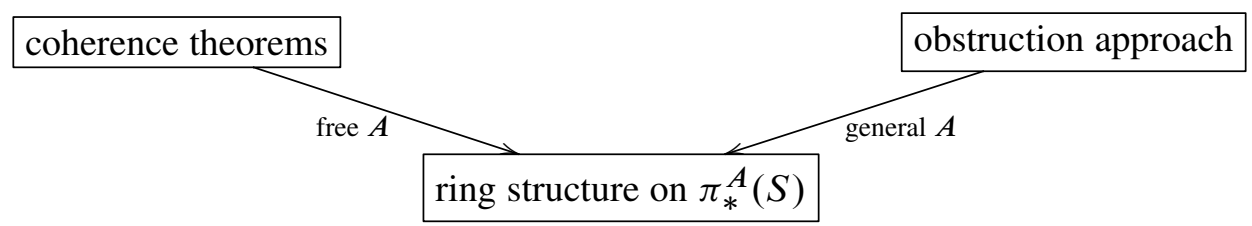

It is natural to wonder if there is some closer connection between the two approaches, but we have been unable to uncover one.

\subsection{Some background and an apology}

From a certain perspective this paper is entirely on the subject of getting signs correct, although the "signs" are not just \pm 1 but rather elements in the 2-torsion of $\operatorname{Aut}(S)$, where $(\mathcal{C}, \otimes, S)$ is a symmetric monoidal category. Many of the results are undoubtedly folklore, but just lacking a convenient reference. Since this is a subject where it seems particularly important to have a convenient reference - no one likes to think about signs - we have included quite a bit of exposition (perhaps overdoing it on occasion).

Associativity and commutativity for $R O(G)$-graded stable homotopy rings have typically been dealt with in a different way than what we describe here. In essence, the 
various choices for isomorphisms are built into the framework from the very beginning, and one is tasked with keeping track of them. We refer the reader to May [14, Chapter 13, Section 1] and May and Sigurdsson [15, Section 21.1] for detailed discussions. After the present paper was released, Mike Mandell kindly informed us that these issues for $R O(G)$-graded rings are also treated in the appendix of Lewis and Mandell [12], by a method that is essentially the same as what we do in Section 7. Finally, it is informative to have a look at Adams [1, Section 6], which shows that these questions go back to the very early days of equivariant topology.

Certainly Proposition 1.2 and related results are if not well known then at least not surprising, although I wonder if the sign in the multiplicativity of the forgetful map (see Proposition 1.19) has been noticed before, either in the motivic or equivariant context. I have also been unable to find a reference in the literature similar to Proposition 6.11, even though the sign questions dealt with by that result are ubiquitous.

Invertible objects are well studied in the literature (for example, in Fröhlich and Wall [6]), but in somewhat sporadic places, and there seem to be some gaps. For example, given a self-map $f: X \rightarrow X$ where $X$ is invertible there are two ways to obtain an element of $\operatorname{End}(S)$. One is called the trace of $f$, and the other is something that does not have a standard name; in this paper we call it the $D$-invariant. These two invariants can be different, although they sometimes get confused. We attempt to give a careful treatment in Section 4.

As far as the coherence statements are concerned, the earliest result along these lines seems to be [3, Lemma 1.4.3], due to Deligne. However, Deligne's result (stated without proof) only applied to symmetric monoidal categories where the self-twists $X \otimes X \rightarrow X \otimes X$ are all equal to the identity; as is clear from the results listed above, this omits the important and nontrivial phenomena that occur in the general case. Symmetric monoidal categories in which all objects are invertible are treated again in [6]. A classification theorem is given (see [6, Corollary 6.6]), from which coherence results are easily deducible, but again only in the case where the self-twists are all equal to the identity. Another sort of classification theorem for such categories is given in the unpublished $\mathrm{PhD}$ thesis [7, Chapter II, Section 2, Proposition 5]; but although Hoàng's Theorem allows for nonidentity self-twists the classification is of a different nature and does not seem to yield any coherence results. The excellent and influential paper [9] by Joyal and Street has coherence results in the braided context, but not for invertible objects; it gives a classification theorem for braided monoidal categories where all objects are invertible, but again not yielding coherence results in any evident way. The literature contains many more sophisticated coherence results than the ones presented here, and so it seems to be merely an unfortunate accident that there is no convenient reference for them. 
Symmetric monoidal categories in which all maps are isomorphisms and all objects are invertible are nowadays often called symmetric categorical groups (or Picard groupoids, in older literature). Our "coherence with twists" may be interpreted in this context (whereas the one-object case of "coherence without twists" is really a coherence theorem for nonsymmetric categorical groups). The coherence theorems are tantamount to determining the "free symmetric categorical group", or "free Picard groupoid", on a given set of objects. After writing this paper it was pointed out to us by Nick Gurski that a different proof, in the case of one object, is given by Johnson and Osorno in [8, Section 3]; see also Remark 4.21 below.

\subsection{Organization of the paper}

In Section 2 we give a brief review of Mac Lane's coherence theorem for monoidal categories, and explain how it gives rise to associativity results for $\mathbb{N}$-graded morphism groups. Section 3 then reviews the deeper coherence theorem of Kelly-Laplaza, which applies to symmetric monoidal categories with left duals. Section 4 develops the basic theory of invertible objects, in particular establishing that the trace of the identity map on such an object has order at most two; this is a key result used throughout the paper. In Section 5 we prove the coherence theorems for invertible objects, and in Section 6 we give the applications to $\mathbb{Z}^{n}$-graded morphism rings. Finally, Section 7 deals with the topic of grading morphism rings by nonfree abelian groups.

Acknowledgements The author is grateful to Nick Gurski, Sharon Hollander, Peter May, Victor Ostrik and Vadim Vologodsky for helpful conversations, and to the anonymous referee for both a careful reading and thoughtful suggestions. This research was partially supported by NSF grant DMS-0905888.

\section{Review of Mac Lane's coherence}

Here we recall how Mac Lane's classical coherence theorem for symmetric monoidal categories gives rise to an associativity result for $\mathbb{N}^{n}$-graded morphism rings.

Let $(\mathcal{C}, \otimes, S)$ be a symmetric monoidal category. Where needed, we will denote the associativity isomorphism $(x \otimes y) \otimes z \cong x \otimes(y \otimes z)$ by $a$ and the symmetry isomorphism $x \otimes y \cong y \otimes x$ by $t$.

Let $w$ be any tensor word made up of formal variables $x_{i}$; for instance, the word $\left(\left(x_{1} \otimes x_{2}\right) \otimes x_{1}\right) \otimes\left(x_{2} \otimes x_{3}\right)$ is one example. Such tensor words can be identified with certain kinds of functors $\mathcal{C}^{n} \rightarrow \mathcal{C}$, where $n$ is the number of letters in the word. 
Precisely, these are the functors that can be built up from $\otimes: \mathcal{C}^{2} \rightarrow \mathcal{C}$ using composition and the operation of cartesian product with identity maps.

Using the associativity and commutativity isomorphisms, there is a "formal isomorphism" (or natural isomorphism) between $w$ and some word $\underline{x}^{a}$, for a uniquely determined $a \in \mathbb{N}^{n}$. To fix such an isomorphism, here is what we do. First, relabel the $x_{1}$ 's in $w$ as $x_{1 a}, x_{1 b}, x_{1 c}$, etc, with the indices appearing alphabetically from left to right in the word. Do the same for all the other $x_{i}$ 's. Let $w^{\prime}$ denote the new word thus constructed. Regard $w^{\prime}$ as a functor $\mathcal{C}^{N} \rightarrow \mathcal{C}$, where $N$ is the total number of variables in $w^{\prime}$. Using the associativity, commutativity, and unital isomorphisms one can construct a natural isomorphism between the functor corresponding to $w^{\prime}$ and the functor corresponding to the word

$$
\left(x_{1 a} \otimes x_{1 b} \otimes \cdots\right) \otimes\left(x_{2 a} \otimes x_{2 b} \otimes \cdots\right) \otimes \cdots \otimes\left(x_{n a} \otimes x_{n b} \otimes \cdots\right) .
$$

Moreover, Mac Lane's coherence theorem [13, Theorem XI.1.1] says that this natural isomorphism is uniquely determined. The exact choices of associativity and commutativity isomorphisms used to construct it are definitely not unique, but the composite isomorphism itself is unique. Now evaluate this natural isomorphism in the case where all the $x_{1, *}$ objects are equal to $x_{1}$, all the $x_{2, *}$ objects are equal to $x_{2}$, etc. This is our definition of $\phi: w \stackrel{\cong}{\cong} \underline{x}^{a}$.

Using the observation of the last paragraph, for $a, b \in \mathbb{N}^{n}$ we obtain canonical isomorphisms

$$
\phi_{a, b}: \underline{x}^{a} \otimes \underline{x}^{b} \stackrel{\cong}{\rightarrow} \underline{x}^{a+b}
$$

such that the following diagram commutes:

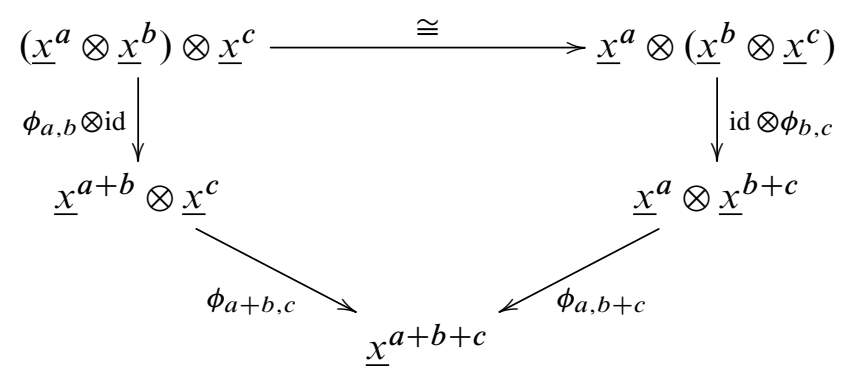

The reason it commutes is again by the coherence theorem. Both ways of moving around the diagram are instances of a natural transformation made up of the associativity and commutativity isomorphisms, where one relabels the $x_{1}$ 's appearing as $x_{1 a}, x_{1 b}$, etc, and the same for the other variables. Mac Lane's Theorem says that there is a unique such natural transformation, and so the two ways of moving around the diagram 
must be the same. (Note also that when $a$ or $b$ is the zero vector then $\phi_{a, b}$ is the unital isomorphism from the symmetric monoidal structure).

Now assume that $\mathcal{C}$ is also an additive category, and that the tensor product is an additive functor in each variable. Let $X_{1}, \ldots, X_{n}$ be fixed objects in $\mathcal{C}$. Consider the $\mathbb{N}^{n}$-graded abelian group

$$
R=\bigoplus_{a \in \mathbb{N}^{n}} \mathrm{C}\left(\underline{X}^{a}, S\right) .
$$

We will also write $R_{a}$ for $\mathcal{C}\left(\underline{X}^{a}, S\right)$. We claim that $R$ has the structure of an $\mathbb{N}^{n}$ graded ring. The product is defined as follows. If $f \in R_{a}$ and $g \in R_{b}$, define $f g \in R_{a+b}$ to be the composition

$$
\underline{X}^{a+b} \stackrel{\phi_{a, b}^{-1}}{\longrightarrow} \underline{X}^{a} \otimes \underline{X}^{b} \stackrel{f \otimes g}{\longrightarrow} S \otimes S \cong S .
$$

2.2 Remark Note that the above product can also be described as the composition

$$
\underline{X}^{a+b} \cong \underline{X}^{a} \otimes \underline{X}^{b} \stackrel{\mathrm{id} \otimes g}{\longrightarrow} \underline{X}^{a} \otimes S \stackrel{f \otimes \mathrm{id}}{\longrightarrow} S \otimes S \cong S .
$$

In this way the product in $R$ can be thought of as induced by the composition in the category $\mathrm{C}$ : $f g$ comes from composing $f$ with an appropriately "suspended" version of $g$.

2.3 Proposition $R$ is a graded ring (associative and unital), and $R_{0}$ is central.

Proof Distributivity follows immediately from the fact that $\otimes$ is biadditive: for instance, if $f, g \in R_{a}$ and $h \in R_{b}$ then the map $(f+g) \otimes h$ is equal to $(f \otimes h)+(g \otimes h)$. So the same remains true when we precompose both with $\phi_{a, b}^{-1}$.

Associativity follows, by an easy argument, from the fact that diagram (2.1) is commutative. The fact that $\phi_{a, b}$ equals the unital isomorphism when $a$ or $b$ is zero implies that the identity element $\operatorname{id}_{S} \in R_{0}$ is a unit for $R$.

For the centrality of $R_{0}$, let $f: S \rightarrow S$ and let $g: \underline{X}^{a} \rightarrow S$. The following diagram is commutative:

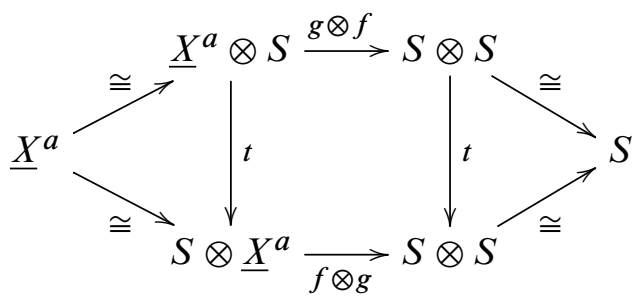

The composition across the top is $f \cdot g$, and across the bottom is $g \cdot f$. 
2.4 Remark This section serves as the prototype for what will happen in the rest of the paper. In the case where the objects $X_{i}$ are invertible under the tensor product, we wish to extend $R$ to a $\mathbb{Z}^{n}$-graded ring. This requires extending the construction of the $\phi_{a, b}$ 's, which in turn depends on a more sophisticated version of coherence.

\section{Kelly-Laplaza coherence}

Let $\mathcal{U}$ be a set. Kelly and Laplaza [11] describe the "free symmetric monoidal category with left duals" on the set $\mathcal{U}$, denoted here $\mathrm{KL}(\mathcal{U})$. In this section we review this construction.

\subsection{Preliminaries}

Let $(\mathcal{C}, \otimes, S)$ be a symmetric monoidal category, and let $X$ be an object. Recall that a left dual for $X$ is an object $Y$ together with maps $\eta: S \rightarrow Y \otimes X$ and $\hat{\eta}: X \otimes Y \rightarrow S$ such that the composites

$$
\begin{gathered}
X=X \otimes S \stackrel{\text { id } \otimes \eta}{\longrightarrow} X \otimes Y \otimes X \stackrel{\hat{\eta} \otimes \mathrm{id}}{\longrightarrow} S \otimes X=X, \\
Y=S \otimes Y \stackrel{\eta \otimes \mathrm{id}}{\longrightarrow} Y \otimes X \otimes Y \stackrel{\text { id } \otimes \hat{\eta}}{\longrightarrow} Y \otimes S=Y,
\end{gathered}
$$

are the respective identities (we are not bothering to write the associativity isomorphisms in the composites, even though they are there). To give an object $Z$ the structure of a left dual of $X$ is the same as giving the functor $Z \otimes(-)$ the structure of a right adjoint to $X \otimes(-)$. This observation makes it clear that if $(Y, \eta, \hat{\eta})$ and $\left(Y^{\prime}, \eta^{\prime}, \hat{\eta}^{\prime}\right)$ are both left duals for $X$ then there is a unique isomorphism $Y \rightarrow Y^{\prime}$ that is compatible with the extra structure.

3.2 Definition A symmetric monoidal category with left duals is a symmetric monoidal category $(\mathcal{C}, \otimes, S)$ together with an assignment $X \mapsto\left(X^{*}, \eta_{X}, \hat{\eta}_{X}\right)$ that equips every object of $\mathcal{C}$ with a left dual. (Warning: note that $\left(X^{*}\right)^{*}$ need not equal $X$, although they will be isomorphic.)

In the above setting, there is a unique way of making $X \rightarrow X^{*}$ into a contravariant functor. This is not included as part of the definition only to minimize the number of things that need to be checked in applications. We will not need the functoriality of duals.

3.3 Example Let $\mathcal{C}$ be the category of finitely-generated vector spaces over a given field $k$, with the usual tensor product. For a vector space $V$ let $V^{*}=\operatorname{Hom}(V, k)$ and 
let $\hat{\eta}_{V}: V \otimes V^{*} \rightarrow k$ be the evaluation map. Let $\eta_{V}: k \rightarrow V^{*} \otimes V$ be the coevaluation map, sending 1 to $\sum_{i} e_{i}^{*} \otimes e_{i}$, where $\left\{e_{i}\right\}$ is some choice of basis for $V$ and $\left\{e_{i}^{*}\right\}$ is the dual basis. It is routine to check that $\eta_{V}$ does not depend on the choice of basis, and that these structures make $\mathcal{C}$ into a symmetric monoidal category with left duals.

Suppose $X$ has a left dual and $f: X \rightarrow X$ is a map. Then we may form the composite

$$
S \stackrel{\eta}{\rightarrow} X^{*} \otimes X \stackrel{\mathrm{id} \otimes f}{\rightarrow} X^{*} \otimes X \stackrel{t}{\rightarrow} X \otimes X^{*} \stackrel{\hat{\eta}}{\rightarrow} S,
$$

and this composite is called the trace of $f$. The uniqueness of left duals (up to isomorphism) shows that $\operatorname{tr}(f)$ is not dependent on the choice of left dual for $X$. A detailed study of more abstract traces in monoidal categories (which in particular includes the present example) can be found in Joyal, Street and Verity [10].

The trace satisfies the following properties.

3.4 Proposition Let $\mathcal{C}$ be a symmetric monoidal category with left duals.

(a) If there is a commutative diagram

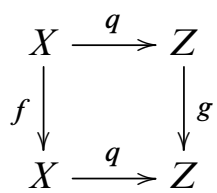

in which $q$ is an isomorphism, then $\operatorname{tr}(f)=\operatorname{tr}(g)$.

(b) If $f: X \rightarrow Y$ and $g: Y \rightarrow X$ then $\operatorname{tr}(f g)=\operatorname{tr}(g f)$.

Proof For part (a), observe that if $\left(X^{*}, \eta, \hat{\eta}\right)$ is a left dual for $X$ then $X^{*}$ is also a left dual for $Z$ via the maps $\eta^{\prime}=\left(\operatorname{id}_{X^{*}} \otimes q\right) \eta$ and $\hat{\eta}^{\prime}=\hat{\eta}\left(q^{-1} \otimes \operatorname{id}_{X^{*}}\right)$. Using this, (a) is an easy exercise.

Part (b) is a little harder. While not really needed in the present paper, we include a sketch for expository purposes; see also [11, Proposition 6.2] and Ponto and Shulman [16, Proposition 2.4]. The point is simply the commutativity of the following diagram:

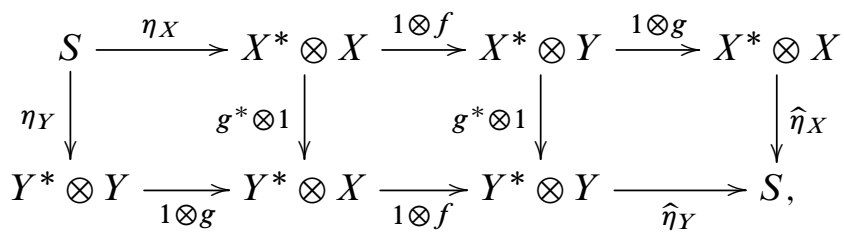


where $g^{*}$ is defined to be

$$
Y^{*} \stackrel{\cong}{\longrightarrow} S \otimes Y^{*} \stackrel{\eta_{X} \otimes 1}{\longrightarrow} X^{*} \otimes X \otimes Y^{*} \stackrel{1 \otimes g \otimes 1}{\longrightarrow} X^{*} \otimes Y \otimes Y^{*} \stackrel{1 \otimes \hat{\eta}_{Y}}{\longrightarrow} X^{*} \otimes S \stackrel{\cong}{\longrightarrow} X^{*} .
$$

The commutativity of the left and right rectangles are easy exercises using the definition of left dual.

We will also need the following fundamental result, which is a form of the classic Eckmann-Hilton argument; see also [11, Proposition 6.1].

3.5 Lemma In a monoidal category the monoid $\operatorname{End}(S)$ is abelian.

Proof Suppose $f, g: S \rightarrow S$ and consider the following commutative diagram:

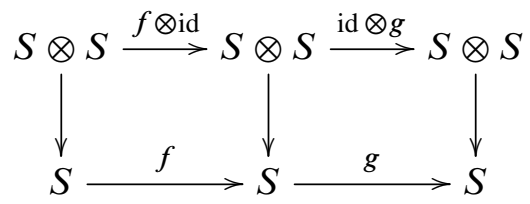

This shows that $g f$ is the composite

$$
S \stackrel{f \cong g}{\longrightarrow} S \otimes S \stackrel{f \cong}{\longrightarrow} S
$$

A similar diagram also shows that $f g$ is also equal to this composite, so $g f=f g$.

\subsection{The construction}

For any category $\mathcal{U}$ the paper [11] constructs the free symmetric monoidal category with left duals on $\mathcal{U}$. We will only need this construction where $\mathcal{U}$ only has identity maps, ie $\mathcal{U}$ is just a set; see Remark 3.8, however, for hints about the general case.

Note that for every element $X \in \mathcal{U}$ our category must have an identity map $\mathrm{id}_{X}$ and therefore a self-map of $S$ obtained by taking the trace. These traces will all need to commute, since all self-maps of $S$ commute by Lemma 3.5. So let $\mathbb{N}\langle\mathcal{U}\rangle$ be the free commutative monoid on the set $\mathcal{U}$. If $X \in \mathcal{U}$ we think of the element $[X] \in \mathbb{N}\langle\mathcal{U}\rangle$ as the formal trace of the identity map on $X$. Our construction for KL(U) will have $\mathbb{N}\langle\mathcal{U}\rangle$ as its set of self-maps of $S$.

Define a signed set to be a set $A$ together with a function $\tau: A \rightarrow\{+,-\}$. If $A$ is a signed set let $A^{*}$ be the same set but with the signs reversed. If $A$ and $B$ are signed sets then $A \amalg B$ denotes the disjoint union with the evident signs. A bipartition of a signed set $A$ is a directed graph with $A$ as the vertex set, having the properties that 
(i) the tail of every edge is marked with - and the head of every edge is marked with + ,

(ii) every element of $A$ is a vertex of exactly one edge.

If $A$ and $B$ are signed sets, then a correspondence from $A$ to $B$ is a bipartition of $A^{*} \amalg B$. One can make a picture of such a thing by drawing the elements of $A$ on one "level", the elements of $B$ on a lower level, and then drawing the edges of the bipartition. For example:
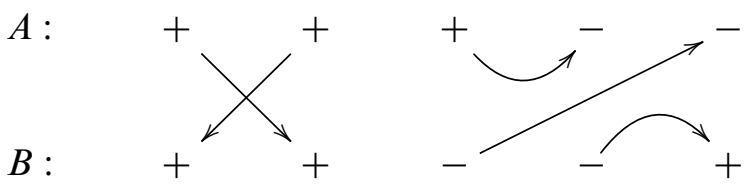

Note the convention for drawing edges with vertices at the same level: if the vertices are on the top level we draw a cup $\cup$, and if the vertices are on the bottom level we draw a cap $\cap$. Also, there is a simple technique for getting the direction of the arrows straight: each element with sign + should be pictured as a small downward arrow $\downarrow$, and elements with sign - are pictured as small upward arrows $\uparrow$. These small arrows must join (compatibly) with the edges in the correspondence. Finally, note again that the data in these pictures is really just "what connects to what". The exact physical paths of the arrows in the picture are irrelevant, only where the arrows begin and end.

Given a correspondence from $A$ to $B$, and a correspondence from $B$ to $C$, we may compose these to get a correspondence from $A$ to $C$. This is best described in terms of the pictures: one stacks the pictures on top of each other and composes the edges head to tail as expected. Note that there might be extra "loops" in the picture, and these must be discarded. For example, the composition

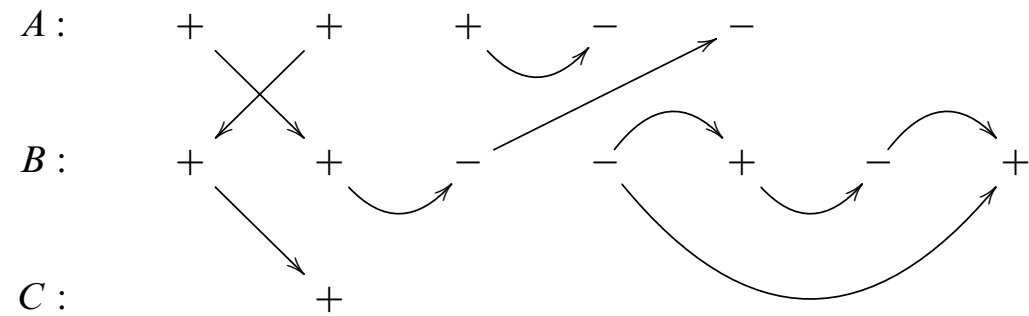

equals the correspondence

$A$ :

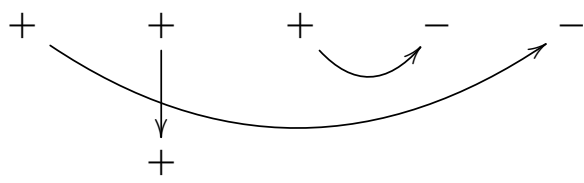

$C$ : 
We are ready to define the category $\mathrm{KL}(\mathcal{U})$. An object will be a formal word made from the set $\mathcal{U}$ and the special symbol $S$ using tensors and duals: eg, $w=\left(\left(X^{*}\right)^{*} \otimes S\right) \otimes$ $\left((X \otimes Y)^{*} \otimes\left(Y^{*} \otimes Z\right)\right)$. To each such word we associate its underlying set of letters $P(w)$, together with a function $\tau: P(w) \rightarrow\{+,-\}$. In the above example $P(w)=\left\{X_{1}, X_{2}, Y_{1}, Y_{2}, Z\right\}$ (the indices distinguish the different occurrences of the letters in the word) and the sign function has $\tau^{-1}(+)=\left\{X_{1}, Z\right\}, \tau^{-1}(-)=$ $\left\{X_{2}, Y_{1}, Y_{2}\right\}$. In general $P$ is defined inductively by setting $P(X)=\{X\}$ if $X \in \mathcal{U}$, $P(S)=\varnothing, P(u \otimes v)=P(u) \amalg P(v)$, and $P\left(u^{*}\right)=P(u)^{*}$. Note that there is an evident map $P(w) \rightarrow \mathcal{U}$ that sends each formal symbol to the corresponding element of $\mathcal{U}$.

Let $w_{1}$ and $w_{2}$ be two formal words. We define a map from $w_{1}$ to $w_{2}$ to be a pair $(\theta, \lambda)$ where $\theta$ is a bipartition of $P\left(w_{1}\right)^{*} \amalg P\left(w_{2}\right)$ for which the head and tail of every edge are sent to the same object of $\mathcal{U}$, and where $\lambda$ is an element of $\mathbb{N}\langle\mathcal{U}\rangle$. Given a map $(\theta, \lambda): w_{1} \rightarrow w_{2}$ and $(\phi, \mu): w_{2} \rightarrow w_{3}$, the composite is $\left(\phi \theta, \lambda+\mu+\sum_{i}\left[X_{i}\right]\right)$ where $\phi \theta$ is the composition of correspondences and where the $X_{i}$ 's are the objects labelling each of the loops that was discarded during the composition process. Said differently, every loop in which the vertices were labelled by an object $X \in \mathcal{U}$ contributes a factor of $\operatorname{tr}\left(\operatorname{id}_{X}\right)=[X]$ to the composition.

We may again depict maps in $\mathrm{KL}(\mathcal{U})$ via pictures. For example, here is a map from $\left(X^{*}\right)^{*} \otimes(X \otimes Y)^{*} \otimes(Z \otimes Y)$ to $Z$ :
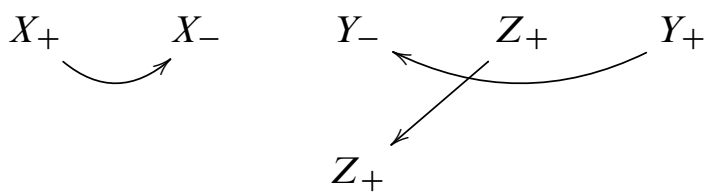

Note that the precise word forming the domain (or codomain) of the map is not retrievable from the picture; that is, the picture only shows $P(w)$ together with a linear ordering, not $w$ itself. This is actually a feature rather than a bug! If two words differ only in the placement of parentheses, for example, notice that there is a canonical isomorphism between them. Similarly, observe that $\left(w^{*}\right)^{*}$ is canonically isomorphic to $w$, and $\left(w_{1} \otimes w_{2}\right)^{*}$ is canonically isomorphic to $w_{1}^{*} \otimes w_{2}^{*}$.

The category $\operatorname{KL}(\mathcal{U})$ is a symmetric monoidal category with left duals. We leave the reader the (not difficult, but informative) exercise of checking this and identifying the necessary structures. The main result of [11] is the following.

3.7 Theorem (Kelly-Laplaza coherence theorem) The category $\operatorname{KL}(\mathcal{U})$ is the free symmetric monoidal category with left duals on the set $\mathcal{U}$. 
3.8 Remark The paper [11] actually describes the free symmetric monoidal category with left duals on a category $\mathcal{A}$. We have only discussed the case where $\mathcal{A}$ is discrete (ie, only has identity maps) because this is all we need for our present purposes. The general case is not very different, however. A map in $\operatorname{KL}(\mathcal{A})$ is a correspondence equipped with a labelling of the edges by maps in $\mathcal{A}$, having the property that if an edge has head $Y$ and tail $X$ then the label belongs to $\operatorname{Hom}_{\mathcal{A}}(X, Y)$. When composing labelled correspondences one composes the labels in the evident manner. Finally, the monoid of formal traces $\mathbb{N}\langle\mathcal{U}\rangle$ must be replaced by something more complex: every self-map in $\mathcal{A}$ must have a formal trace, and these must satisfy the cyclic property of Proposition 3.4(b). It is easy to write down the universal monoid having these properties; see [11] for details.

\subsection{Uses of coherence}

Now let $\mathcal{C}$ be a symmetric monoidal category with left duals. Let $\mathcal{U} \subseteq$ ob(C) be a set of objects. The Kelly-Laplaza coherence theorem says that there is a map of symmetric monoidal categories $F: \operatorname{KL}(\mathcal{U}) \rightarrow \mathcal{C}$ sending the formal word $[X]$ to $X$, and the formal word $[X]^{*}$ to $X^{*}$, for each $X \in \mathcal{U}$. The functor $F$ also has the following behavior:

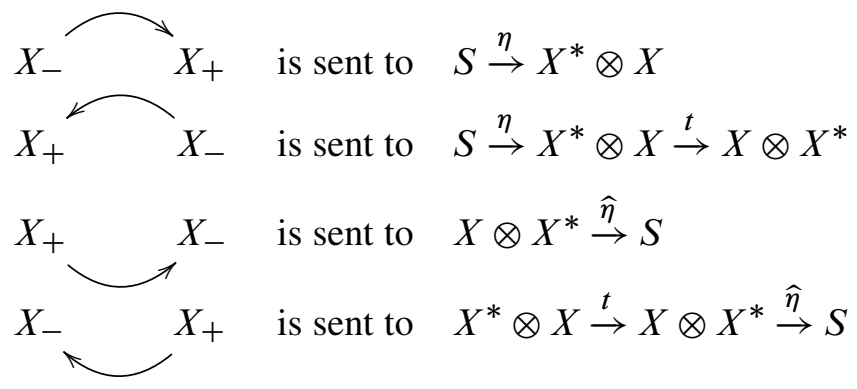

Note: It is worth taking time to think about the second and fourth cases; these composites are also represented by other pictures in which there is a crossing between the edges, but in $\operatorname{KL}(\{\mathcal{U}\})$ such pictures represent the same maps as what we have given; remember that the only part of the pictures that matters is "what connects to what".

As an example, suppose that $X$ and $Y$ are dualizable objects in $\mathcal{C}$ with chosen duals $X^{*}$ and $Y^{*}$. Consider the following two maps from $X \otimes Y \otimes X^{*} \otimes Y^{*} \otimes Y$ to $Y$ :

$$
\begin{gathered}
X Y X^{*} Y^{*} Y \rightarrow Y X X^{*} Y^{*} Y \rightarrow Y S Y^{*} Y=Y Y^{*} Y \rightarrow S Y=Y \\
X Y X^{*} Y^{*} Y \rightarrow X X^{*} Y Y^{*} Y \rightarrow S Y S=Y
\end{gathered}
$$

(We have suppressed the tensor symbols and associativity maps; each of the displayed maps is the evident one that uses the symmetric monoidal structure and the duality maps.) Are the maps in (3.10) and (3.11) guaranteed to be the same in $\mathcal{C}$ ? We work 
in $\operatorname{KL}(\{X, Y\})$ and note that the two composites are represented by the following pictures:
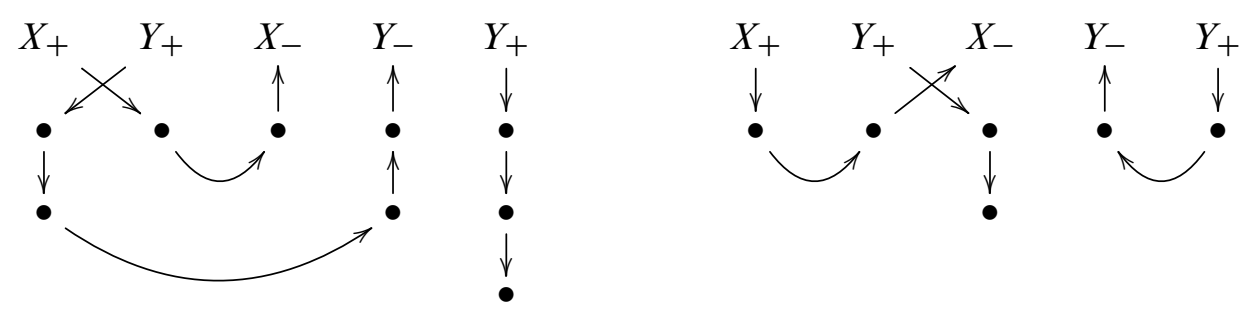

The composite pictures are clearly not the same map in $\operatorname{KL}(\mathcal{U})$, and so there is no guarantee that the two maps are the same in $\mathcal{C}$. They might be the same, but if so this is an "accident;" it does not follow from the basic axioms.

As one more example, let us consider the following composite:

$$
\begin{array}{r}
S=S S S \longrightarrow X^{*} X Y^{*} Y X X^{*} \longrightarrow X^{*} Y^{*} X X Y X^{*} \stackrel{t_{X, X}}{\longrightarrow} X^{*} Y^{*} X X Y X^{*} \\
S=S S S \longleftarrow X^{*} X Y^{*} Y X X^{*}
\end{array}
$$

Note that in the third map we have omitted the identity factors on either side of the $t_{X, X}$, due to limitations of space. All of the other maps are the evident ones. We claim that the composite can be given a simpler description. Computing in $\operatorname{KL}(\{X, Y\})$ we get the following picture:

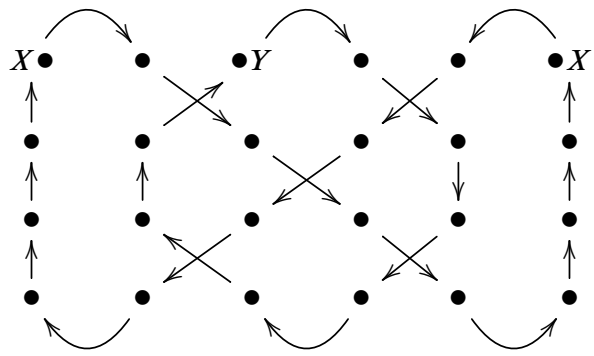

This picture breaks up into two loops, one where the vertices are all labelled by $X$ and the other where they are all labelled by $Y$. As a map from $S$ to $S$ in $\operatorname{KL}(\{X, Y\})$ this composite is therefore equal to $\operatorname{tr}\left(\operatorname{id}_{X}\right) \circ \operatorname{tr}\left(\operatorname{id}_{Y}\right)$ (note that the order of composition does not matter, since $\operatorname{Hom}(S, S)$ is commutative). Since this identity holds in the universal example $\operatorname{KL}(\{X, Y\})$, it also holds in $\mathcal{C}$.

3.12 Remark (Traces in Kelly-Laplaza categories) Let $w$ be an object in KL(\{U\}) and let $f: w \rightarrow w$ be a map. Then $\operatorname{tr}(f)$ is a map $S \rightarrow S$ in KL(\{U\}). We leave it as an easy exercise to verify that $\operatorname{tr}(f)$ is represented by the following picture: 


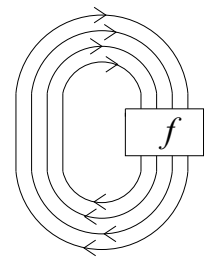

(where the picture representing $f$ should be inserted into the blank box).

\section{Invertible objects}

In this section we review the notion of invertible object in a symmetric monoidal category, and establish some of their properties. For every invertible object $X$ we define a basic commuter $\tau_{X}$, which is an isomorphism $\tau_{X}: S \rightarrow S$ such that $\tau_{X}^{2}=1$.

\subsection{Prelude}

Throughout this section $(\mathcal{C}, \otimes, S)$ is a symmetric monoidal category. If $u: S \rightarrow S$ and $g: A \rightarrow B$ then denote the composites

$$
A \cong S \otimes A \stackrel{u \otimes g}{\longrightarrow} S \otimes B \cong B \text { and } A \cong A \otimes S \stackrel{g \otimes u}{\longrightarrow} B \otimes S \cong B
$$

by $u \hat{\otimes} g$ and $g \hat{\otimes} u$. We will sometimes omit the carat and just write $u \otimes g$ and $g \otimes u$ by abuse, but at other times it is useful to remember that $u \hat{\otimes} g$ and $u \otimes g$ are somewhat different.

4.2 Lemma Let $u: S \rightarrow S$ and $g: A \rightarrow B$. Then

$$
u \hat{\otimes} g=\left(u \hat{\otimes} \mathrm{id}_{B}\right) \circ g=g \circ\left(u \hat{\otimes} \mathrm{id}_{A}\right)=g \hat{\otimes} u .
$$

Proof This is elementary, using that $u \otimes g=\left(\mathrm{id}_{S} \otimes g\right) \circ\left(u \otimes \mathrm{id}_{A}\right)=\left(u \otimes \mathrm{id}_{B}\right) \circ$ $\left(\mathrm{id}_{S} \otimes g\right.$ ) and similarly for $g \otimes u$.

4.3 Remark The above lemma will often be used in the following way. Suppose that $g: A \rightarrow B$ and $f: B \rightarrow C$. Then multiple applications of the lemma give

$$
(u \hat{\otimes} f) \circ g=f \circ\left(u \hat{\otimes} \operatorname{id}_{B}\right) \circ g=f \circ(u \hat{\otimes} g)=f \circ g \circ\left(u \hat{\otimes} \operatorname{id}_{A}\right)=u \hat{\otimes}(f g) .
$$

So we can move a $u \hat{\otimes}(-)$ from anywhere inside a composite to anywhere else, including outside the composite.

4.4 Remark Observe that for any object $V$ in $\mathcal{C}$ we obtain a map of monoids $\operatorname{End}(S) \rightarrow \operatorname{End}(V)$ given by $u \mapsto u \widehat{\otimes} \mathrm{id}_{V}$. 


\subsection{Invertible objects}

4.6 Definition An object $X$ in $\mathcal{C}$ is invertible if there exists a $Y$ in $\mathcal{C}$ and an isomorphism $\eta: S \rightarrow Y \otimes X$.

It is easy to prove that if such a $Y$ exists then it is unique up to isomorphism. But to give an inverse for an object $X$, one must specify an object $Y$ together with the isomorphism $\eta: S \rightarrow Y \otimes X$. This map $\eta$ is not uniquely determined by $Y$, since one can clearly get a different $\eta$ by precomposing with an automorphism of $S$. Note that if $X$ and $Z$ are invertible then clearly so is $X \otimes Z$.

We will often use the following observation.

4.7 Proposition If $X$ is an invertible object in $\mathcal{C}$, then we have that the canonical map $\operatorname{End}(S) \rightarrow \operatorname{End}(X)$ is an isomorphism of monoids. More generally, for any object $V$ of $\mathrm{C}$ the two maps $\operatorname{End}(V) \rightarrow \operatorname{End}(V \otimes X)$ and $\operatorname{End}(V) \rightarrow \operatorname{End}(X \otimes V)$ (obtained by tensoring with identity maps) are both isomorphisms.

Proof Choose an inverse $(Y, \eta)$ for $X$. The functor $T_{X}: \mathcal{C} \rightarrow \mathcal{C}$ given by $Z \mapsto Z \otimes X$ is an equivalence of categories, because an inverse is given by $W \mapsto W \otimes Y$. Since $T_{X}$ is an equivalence, for any $V$ in $\mathcal{C}$ the map $\operatorname{End}(V) \rightarrow \operatorname{End}\left(T_{X}(V)\right)$ is an isomorphism of monoids. A similar argument shows $\operatorname{End}(V) \rightarrow \operatorname{End}(X \otimes V)$ to be an isomorphism (or use the twist map $X \otimes V \cong V \otimes X$ ).

When $V=S$ one has $T_{X}(S) \cong X$ via the unital isomorphism, and the composite $\operatorname{End}(S) \rightarrow \operatorname{End}\left(T_{X}(S)\right) \cong \operatorname{End}(X)$ is readily checked to be the map of Remark 4.4.

When $X$ is invertible it will be useful to have a description of the inverse to the isomorphism $\operatorname{End}(S) \rightarrow \operatorname{End}(X)$. If $(Y, \eta)$ is a choice of inverse for $X$ and $g: X \rightarrow X$, define $D_{Y}(g)$ to be the composite

$$
S \stackrel{\eta}{\rightarrow} Y \otimes X \stackrel{\mathrm{id} \otimes g}{\longrightarrow} Y \otimes X \stackrel{\eta^{-1}}{\longrightarrow} S
$$

An easy diagram chase shows that $D_{Y}\left(u \hat{\otimes} \mathrm{id}_{X}\right)=u$ for $u \in \operatorname{End}(S)$. Since the map $\operatorname{End}(S) \rightarrow \operatorname{End}(X)$ is an isomorphism this verifies that $D_{Y}$ is the inverse, and so in particular does not depend on the choice of $(Y, \eta)$. From now on we will just write $D(g)$ rather than $D_{Y}(g)$.

The homomorphism $D: \operatorname{End}(X) \rightarrow \operatorname{End}(S)$ is a bit like a trace, but it does not coincide with the standard trace that exists for dualizable objects as defined in Section 3.1 (see Remark 4.16 for an explicit example). The map $D$ can also be regarded as something 
like a determinant; this analogy works well when $\mathcal{C}$ is a category of vector spaces or vector bundles, but of course in those cases the determinant and trace are indistinguishable on one-dimensional objects. In the present paper we will just call $D(f)$ the " $D$ invariant" of the map $f: X \rightarrow X$. Like a determinant, the $D$-invariant is multiplicative (being a homomorphism of monoids): that is, $D\left(\mathrm{id}_{X}\right)=\mathrm{id}_{S}$ and $D(f g)=D(f) D(g)$. Here are some further properties of the $D$-invariant.

4.8 Lemma Let $X$ and $Z$ be invertible objects.

(a) Given a commutative diagram

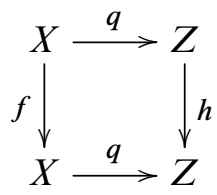

in which $q$ is an isomorphism, one has $D(f)=D(h)$.

(b) If $f: X \rightarrow X$, then $D\left(\operatorname{id}_{Z} \otimes f\right)=D\left(f \otimes \mathrm{id}_{Z}\right)=D(f)$.

(c) If $f: X \rightarrow X$ and $g: Z \rightarrow Z$ then $D(f \otimes g)=D(f) D(g)$, where the product on the right-hand side is in the monoid $\operatorname{End}(S)$.

Proof All of the parts are easy exercises. For (a) one uses the diagram

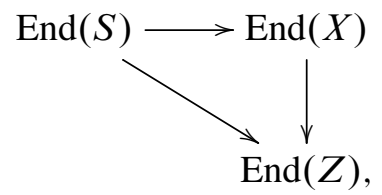

where the vertical arrow sends a map $f$ to $q f q^{-1}$. One checks that the diagram commutes using Remark 4.3, and then it follows at once that $D\left(q f q^{-1}\right)=D(f)$.

For (b) one looks at the composite $\operatorname{End}(S) \rightarrow \operatorname{End}(X) \rightarrow \operatorname{End}(X \otimes Z)$. Both maps are isomorphisms, $D_{X}$ is the inverse of the first map, and $D_{X \otimes Z}$ is the inverse of the composite; it follows at once that $D_{X \otimes Z}\left(f \otimes \mathrm{id}_{Z}\right)=D(f)$.

Finally, (c) follows from (b) and the fact that $f \otimes g=\left(f \otimes \mathrm{id}_{Z}\right) \circ\left(\operatorname{id}_{X} \otimes g\right)$.

4.9 Remark Let $f: A \rightarrow B$ and $g: B \rightarrow B$, where $B$ is invertible. The $D$-invariant of $g$ is the unique map $S \rightarrow S$ satisfying $g=D(g) \hat{\otimes} \mathrm{id}_{B}$. We can then write

$$
g f=\left(D(g) \hat{\otimes} \mathrm{id}_{B}\right) \circ f=f \circ\left(D(g) \hat{\otimes} \mathrm{id}_{A}\right),
$$

using Remark 4.3 for the second equality. So automorphisms of invertible objects can effectively be moved around inside a composition, by replacing them with their $D$-invariant. 


\subsection{The adjoint to $\eta$ and the trace of a map}

The following result shows that an invertible object is left dualizable.

4.11 Proposition Let $X$ be an invertible object in $\mathcal{C}$, with inverse $(Y, \eta)$. Then there is a unique map $\hat{\eta}: X \otimes Y \rightarrow S$ with the property that the composite

$$
X \cong X \otimes S \stackrel{\text { id } \otimes \eta}{\longrightarrow} X \otimes(Y \otimes X) \cong(X \otimes Y) \otimes X \stackrel{\hat{\eta} \otimes \text { id }}{\longrightarrow} S \otimes X \cong X
$$

equals the identity. Moreover, $\hat{\eta}$ is an isomorphism and the composite

$$
Y \cong S \otimes Y \stackrel{\eta \otimes \text { id }}{\longrightarrow}(Y \otimes X) \otimes Y \cong Y \otimes(X \otimes Y) \stackrel{\text { id } \otimes \widehat{\eta}}{\longrightarrow} Y \otimes S \cong Y
$$

also equals the identity.

4.12 Remark Note that one is tempted to assume that $\hat{\eta}$ equals the composite

$$
X \otimes Y \stackrel{t}{\rightarrow} Y \otimes X \stackrel{\eta^{-1}}{\longrightarrow} S .
$$

This need not be the case. Let $k$ be a field and let $\mathcal{C}=$ GrVect $_{k}^{ \pm}$be the category of $\mathbb{Z}$-graded vector spaces with the usual tensor product, and with the twist map that involves signs. Let $X=k[1]$, and $Y=k[-1]$. Let $\eta: k \rightarrow Y \otimes X$ send 1 to $1 \otimes 1$. Then $\hat{\eta}: X \otimes Y \rightarrow k$ must be the multiplication map, whereas the composite (4.13) sends $a \otimes b$ to $-a b$.

Proof of Proposition 4.11 Let the functor $F: \mathcal{C} \rightarrow \mathcal{C}$ be given by $F(A)=X \otimes A$, and let $G: \mathcal{C} \rightarrow \mathcal{C}$ be given by $G(A)=Y \otimes A$. These are an equivalence of categories, and the map $\eta$ gives a natural isomorphism Id $\rightarrow G F$. The proof basically consists of the observation that any equivalence of categories is an adjoint equivalence; moreover, any choice of unit completely determines the corresponding counit by an explicit formula. We are grateful to the referee for providing this perspective.

Given any object $A$ in $\mathcal{C}$, choose an isomorphism $\beta: F G(A) \rightarrow A$ (such an isomorphism exists because $F$ and $G$ are an equivalence). Then the top row of the following diagram is a new isomorphism $F G(A) \rightarrow A$ that turns out to be independent of the choice of $\beta$ :

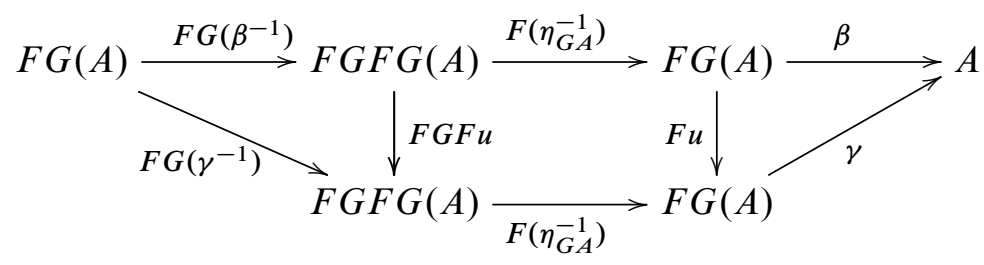


Indeed, if $\gamma: F G(A) \rightarrow A$ is another choice of isomorphism then $\gamma^{-1} \beta$ equals $F(u)$ for a unique $u: G A \rightarrow G A$ (since $F$ is an equivalence). The above diagram is then readily checked to commute, and so the two composites around the outside coincide.

The preceding paragraph shows how to define the counit maps $\hat{\eta}_{A}: F G A \rightarrow A$. It is easy to verify that the composites $G \rightarrow G F G \rightarrow G$ and $F \rightarrow F G F \rightarrow F$ are indeed the respective identities. The only remaining thing to verify is that $\hat{\eta}$ is natural; but given a map $k: A \rightarrow Z$, commutativity of the square

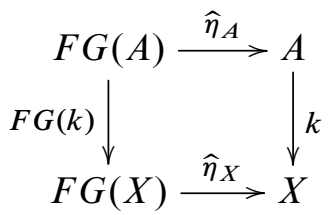

will follow from commutativity of the corresponding diagram obtained by applying $G$. But this in turn may be checked by precomposing with the unit isomorphisms $\eta_{G(-)}: G(-) \rightarrow G F G(-)$ and using the unit/counit identities already established.

4.14 Remark We will not need this, but notice that the above proof provides an explicit construction of $\hat{\eta}: X \otimes Y \rightarrow S$, obtained for example by taking the isomorphism of (4.13) to be the map $\beta: F G(S) \rightarrow S$ that appears in the proof.

Suppose that $X$ is invertible and $f: X \rightarrow X$. Since $X$ is left dualizable we may take the trace, obtaining $\operatorname{tr}(f): S \rightarrow S$. Recall that we have another way to obtain a self-map of $S$, namely the $D$-invariant $D(f)$. These are connected by the following formula.

4.15 Proposition Let $X$ be an invertible object and let $f: X \rightarrow X$. Then one has $\operatorname{tr}\left(\operatorname{id}_{X}\right) \cdot D(f)=\operatorname{tr}(f)$.

Proof The composite $\operatorname{tr}\left(\mathrm{id}_{X}\right) \cdot D(f)$ is

$$
S \stackrel{\eta}{\rightarrow} Y \otimes X \stackrel{\mathrm{id} \otimes f}{\rightarrow} Y \otimes X \stackrel{\eta^{-1}}{\rightarrow} S \stackrel{\eta}{\rightarrow} Y \otimes X \stackrel{\text { id }}{\rightarrow} Y \otimes X \stackrel{t}{\rightarrow} X \otimes Y \stackrel{\widehat{\eta}}{\rightarrow} S .
$$

The terms in the middle cancel and we obtain the definition of $\operatorname{tr}(f)$.

4.16 Remark Consider again the example GrVect ${ }_{k}^{ \pm}$from Remark 4.12, with $X, Y$ and $\eta$ as described there. $\operatorname{Then} \operatorname{tr}\left(\operatorname{id}_{X}\right)=-1$, but of course $D\left(\operatorname{id}_{X}\right)=1$. So this gives an example where the trace and $D$-invariant are distinct. 
4.17 Remark Returning again to Remark 4.12, it is worth noting that the difference between $\hat{\eta}$ and (4.13) is in some sense a main theme of the rest of this paper. These are both isomorphisms $X \otimes Y \rightarrow S$, and their "difference" may be measured by the composite

$$
S \stackrel{\eta}{\rightarrow} Y \otimes X \stackrel{t}{\rightarrow} X \otimes Y \stackrel{\hat{n}}{\rightarrow} S
$$

But this composite is just $\operatorname{tr}\left(\mathrm{id}_{X}\right)$.

Our next major goal will be to prove that when $X$ is invertible one has $\operatorname{tr}\left(\operatorname{id}_{X}\right)^{2}=\mathrm{id}_{S}$. This is an important property of invertible objects, but unfortunately we have not been able to find a direct, simple-minded proof. We will instead deduce the result from a cyclic permutation property, which a priori feels somewhat deeper.

\subsection{Automorphisms of invertible objects induced from permutations}

Let $x_{1}, \ldots, x_{n}$ be formal variables and let $w$ be any tensor word in the $x_{i}$ 's with the property that each $x_{i}$ appears exactly once. For instance, if $n=3$ we might have $w=\left(x_{1} \otimes x_{3}\right) \otimes x_{2}$. We can associate to $w$ a functor $F_{w}$ : $\mathcal{C}^{n} \rightarrow \mathcal{C}$ which plugs in objects for the variables $x_{i}$. For objects $X_{1}, \ldots, X_{n}$ in $\mathcal{C}$ write $w\left(X_{1}, \ldots, X_{n}\right)$ as shorthand for $F_{w}\left(X_{1}, \ldots, X_{n}\right)$, and write $w(X)$ as shorthand for $F_{w}(X, X, \ldots, X)$. If $\sigma$ is a permutation of $\{1, \ldots, n\}$, we let $w \sigma$ denote the word in which $x_{i}$ has been replaced by $x_{\sigma(i)}$. So we can write

$$
F_{w \sigma}\left(X_{1}, \ldots, X_{n}\right)=F_{w}\left(\sigma \cdot\left(X_{1}, \ldots, X_{n}\right)\right)=F_{w}\left(X_{\sigma(1)}, X_{\sigma(2)}, \ldots, X_{\sigma(n)}\right) .
$$

By Mac Lane's coherence theorem [13, Theorem XI.1.1] there is a unique natural transformation $F_{w} \rightarrow F_{w \sigma}$ obtained by composing associativity and commutativity isomorphisms. If $X$ is an object in $\mathcal{C}$, we can evaluate this natural transformation at the tuple $(X, X, \ldots, X)$ and thereby obtain an automorphism $\phi_{w, \sigma}: w(X) \rightarrow w(X)$. In this way we obtain a function $\phi_{w}: \Sigma_{n} \rightarrow \operatorname{Aut}(w(X))$, which is readily checked to be a homomorphism.

The following result is from Voevodsky [17, discussion preceding Theorem 4.3].

4.19 Lemma Let $X$ be an invertible object in $\mathcal{C}$. Then for any tensor word $w$ in $n$ variables, and any even permutation $\sigma$ in $\Sigma_{n}$, the map $\phi_{w, \sigma}: w(X) \rightarrow w(X)$ is equal to the identity. In particular, the composite map

$$
(X \otimes X) \otimes X \stackrel{t_{X \otimes X, X}}{\longrightarrow} X \otimes(X \otimes X) \stackrel{a}{\rightarrow}(X \otimes X) \otimes X
$$

is equal to the identity. (Note that this composite map is an instance of the canonical map $(A \otimes B) \otimes C \rightarrow(C \otimes A) \otimes B$, ie the cyclic permutation map). 
Proof Since $X$ is invertible, so is $w(X)$. Therefore, $\operatorname{Aut}(w(X))$ is abelian by Proposition 4.7 and Lemma 3.5. This means the homomorphism $\phi_{w}: \Sigma_{n} \rightarrow \operatorname{Aut}(w(X))$ kills the commutator subgroup of $\Sigma_{n}$, which is the alternating group $A_{n}$.

We can now obtain our goal.

4.20 Proposition If $X$ is an invertible object then $\operatorname{tr}\left(\mathrm{id}_{X}\right)^{2}=\mathrm{id}_{S}$.

Proof Let $f=\operatorname{tr}\left(\operatorname{id}_{(X \otimes X) \otimes X}\right)$. In the Kelly-Laplaza category $\operatorname{KL}(\{X\})$ this is represented by the picture

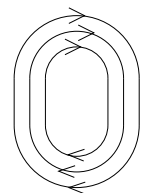

from which one obtains that $f=\operatorname{tr}\left(\operatorname{id}_{X}\right)^{3}$ (due to the three components).

On the other hand, by Lemma $4.19 f$ is also equal to $\operatorname{tr}(c)$, where $c=a \circ t_{X \otimes X, X}$ is the cyclic permutation map. In the Kelly-Laplaza category this is represented by the picture

and from this one obtains $f=\operatorname{tr}\left(\mathrm{id}_{X}\right)$ (due to the one connected component). So we have proven that $\operatorname{tr}\left(\mathrm{id}_{X}\right)^{3}=\operatorname{tr}\left(\mathrm{id}_{X}\right)$.

However, when $X$ is invertible all the maps in the composite defining $\operatorname{tr}\left(\operatorname{id}_{X}\right)$ are isomorphisms; so $\operatorname{tr}\left(\mathrm{id}_{X}\right)$ is an isomorphism. We can therefore cancel one $\operatorname{tr}\left(\mathrm{id}_{X}\right)$ from each side of the previous equation to obtain $\operatorname{tr}\left(\mathrm{id}_{X}\right)^{2}=\mathrm{id}_{S}$.

4.21 Remark In [8, Theorem C] it is proven that the Postnikov 1-truncation of the sphere spectrum represents the free "Picard groupoid" on one object (Picard groupoids are symmetric monoidal categories in which all objects are invertible and all maps are isomorphisms). The stable homotopy group $\pi_{1}(S)=\mathbb{Z} / 2$ therefore represents the endomorphism group of the objects in this free category. This gives another proof of Proposition 4.20, albeit a more abstract one. We are grateful to Nick Gurski for pointing out this reference. 


\subsection{Basic commuters for invertible objects}

If $X$ is an invertible object define $\tau_{X}=\operatorname{tr}\left(\operatorname{id}_{X}\right) \in \operatorname{Aut}(S)$ and call this the basic commuter associated to $X$. These elements will be important in our treatment of skewcommutativity in Section 6. Note that if $X \cong X^{\prime}$ then $\tau_{X}=\tau_{X^{\prime}}$, by Proposition 3.4(a). Let Pic(C) denote the set of isomorphism classes of invertible objects in $\mathcal{C}$; the tensor product makes this set into a group. We have produced a set map $\tau: \operatorname{Pic}(\mathcal{C}) \rightarrow \operatorname{Aut}(S)$, whose image lands in the 2 -torsion subgroup ${ }_{2} \operatorname{Aut}(S)$ by Proposition 4.20.

4.23 Proposition The map $\tau: \operatorname{Pic}(\mathcal{C}) \rightarrow{ }_{2} \operatorname{Aut}(S)$ is a group homomorphism.

Proof We need only show that if $X$ and $Z$ are invertible then $\tau_{X \otimes Z}=\tau_{X} \cdot \tau_{Z}$. For this we work in the Kelly-Laplaza category $\operatorname{KL}(\{X, Z\})$ and observe that the map $\operatorname{tr}\left(\mathrm{id}_{X \otimes Z}\right)$ is represented by the picture

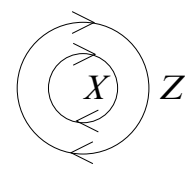

which also represents $\operatorname{tr}\left(\mathrm{id}_{X}\right) \cdot \operatorname{tr}\left(\mathrm{id}_{Z}\right)$.

We may also describe $\tau_{X}$ in terms of the twist map $t_{X, X}: X \otimes X \rightarrow X \otimes X$.

4.24 Proposition $\tau_{X}=\operatorname{tr}\left(\operatorname{id}_{X}\right)=D\left(t_{X, X}\right)=\operatorname{tr}\left(t_{X, X}\right)$

Proof The first equality is the definition. By Proposition 4.15 we have that $\operatorname{tr}\left(\mathrm{id}_{X} \otimes X\right)$. $D\left(t_{X, X}\right)=\operatorname{tr}\left(t_{X, X}\right)$, but $\operatorname{tr}\left(\operatorname{id}_{X \otimes X}\right)=\tau_{X \otimes X}=\tau_{X}^{2}=1$ using Proposition 4.23. This proves the third equality. We can complete the proof by showing that $\operatorname{tr}\left(\mathrm{id}_{X}\right)=\operatorname{tr}\left(t_{X, X}\right)$. This actually follows by the Kelly-Laplaza theorem, for $\operatorname{tr}\left(t_{X, X}\right)$ is represented by the picture below:

Because there is only one connected component, this also represents $\operatorname{tr}\left(\mathrm{id}_{X}\right)$. 


\section{Coherence for invertible objects}

In this section we prove our main coherence theorems for invertible objects in a symmetric monoidal category. We deduce these as consequences of the Kelly-Laplaza theorem.

Let $(\mathcal{C}, \otimes, S)$ be a symmetric monoidal category and let $X$ be an invertible object with inverse $\left(X^{*}, \eta\right)$. Recall that $\operatorname{tr}\left(\operatorname{id}_{X}\right)$ is defined to be the composite

$$
S \stackrel{\eta}{\rightarrow} X^{*} \otimes X \stackrel{t}{\rightarrow} X \otimes X^{*} \stackrel{\hat{n}}{\rightarrow} S
$$

Every map in this composite is an isomorphism, so we can write

$$
\eta^{-1}=\operatorname{tr}\left(\mathrm{id}_{X}\right)^{-1} \circ(\hat{\eta} t)=\operatorname{tr}\left(\mathrm{id}_{X}\right) \circ(\hat{\eta} t)=\operatorname{tr}\left(\mathrm{id}_{X}\right) \hat{\otimes}(\hat{\eta} t),
$$

where in the second equality we have used that $\operatorname{tr}\left(\mathrm{id}_{X}\right)^{2}=\mathrm{id}_{S}$ (see Proposition 4.20). Similarly, we have

$$
\hat{\eta}^{-1}=\operatorname{tr}\left(\mathrm{id}_{X}\right) \hat{\otimes}(t \eta) .
$$

Let $\mathcal{C}^{\text {inv }}$ be the full subcategory of invertible objects. Then $\mathcal{C}^{\text {inv }}$ is a symmetric monoidal category with left duals. We will deduce our desired coherence theorems for invertible objects from Kelly-Laplaza coherence applied to $\mathcal{C}^{\text {inv }}$.

We saw in Section 3 that maps in a Kelly-Laplaza category can be represented by pictures consisting of certain kinds of directed curves in the plane. These curves are very simple: every crossing is a standard " $X$ '"-crossing, and every place where there is a horizontal tangent line is either a local minimum or local maximum with respect to the $y$-coordinate (ie, a cup or cap); we will call these cups and caps the critical points of the curve.

\subsection{Coherence without self-twists}

Proof of Theorem 1.6 Let us use the term "acceptable" for formal composites of the type considered in the statement of the proposition. Suppose there were two acceptable formal composites $f, g: w_{1} \rightarrow w_{2}$ that yielded different maps in $\mathcal{C}$. Then the formal composite $g^{-1} f: w_{1} \rightarrow w_{1}$ would yield a map in $\mathcal{C}$ different from the identity. So it suffices to prove the proposition in the case $w_{1}=w_{2}$ and $g=\mathrm{id}$.

Suppose $f: w_{1} \rightarrow w_{1}$ is an acceptable formal composite such that $f(\mathcal{C}) \neq$ id. Let $w_{1}^{*}$ be the formal inverse of the word $w_{1}$, and choose any acceptable formal composite $h: S \rightarrow w_{1}^{*} \otimes w_{1}$. Consider the formal composite

$$
S \stackrel{h}{\rightarrow} w_{1}^{*} \otimes w_{1} \stackrel{\text { id } \otimes f}{\longrightarrow} w_{1}^{*} \otimes w_{1} \stackrel{h^{-1}}{\longrightarrow} S .
$$


Since $f(\mathcal{C}) \neq$ id Proposition 4.7 shows that the map id $\otimes f$ also does not give the identity in $\mathcal{C}$, and from this it follows that the above composite does not give the identity either. So it suffices to prove the proposition in the case $w_{1}=w_{2}=S$ and $g=$ id.

Let $n_{1}$ be the number of $\eta^{-1}$ maps that appear in $f$, and let $n_{2}$ be the number of $\hat{\eta}^{-1}$ maps that appear. Let $n=n_{1}+n_{2}$. Let $F$ be the formal composite in which every $\eta^{-1}$ has been replaced with $\hat{\eta} t$ and every $\hat{\eta}^{-1}$ has been replaced with $t \eta$. Using Remark 4.3, the identities $\eta^{-1}=\operatorname{tr}\left(\operatorname{id}_{X}\right) \hat{\otimes}(\hat{\eta} t)$ and $\hat{\eta}^{-1}=\operatorname{tr}\left(\operatorname{id}_{X}\right) \hat{\otimes}(t \eta)$ show that $f(\mathcal{C})=\operatorname{tr}\left(\operatorname{id}_{X}\right)^{n} \hat{\otimes} F(\mathcal{C})$.

The reason for introducing $F$ is that it only involves maps that exist for dualizable objects, rather than invertible ones. So we may consider $F$ as a composite in the Kelly-Laplaza category $\operatorname{KL}(\{X\})$. The assumption that $f$ was acceptable implies that $F$ can be represented by the disjoint union of simple closed curves; for example, one of the components might look like this:

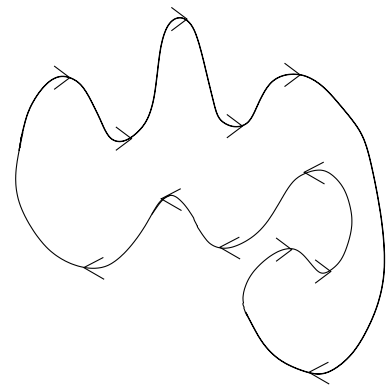

Let us be clear about why this works. The assumption that the formal composite $f$ is acceptable guarantees that the only twist maps that appear in $F$ come together with an $\eta$ or $\hat{\eta}$. In terms of the pictures, each of these twists can be eliminated; to see this, recall how the pictures work:

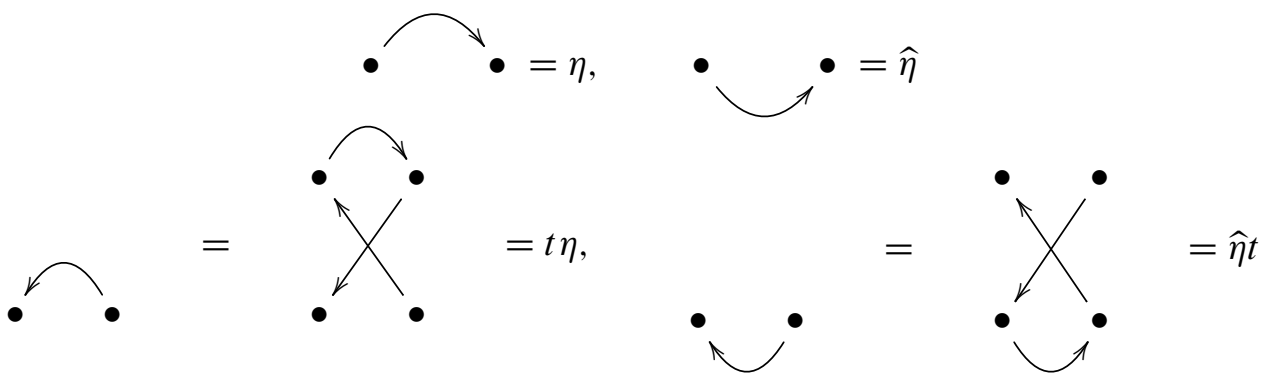

The twists in $F$ only appear in conjunction with a cup or cap, and so they can all be depicted by an untwisted cup or cap going in the opposite direction. 
Now we come to the crux of the matter. For the moment assume that the picture for $F$ only contains one component, for simplicity. In this simple closed curve, every $\eta$ or $\hat{\eta}$ appears as a right-pointing arrow on a cup or cap. Likewise, every $t \eta$ or $\hat{\eta} t$ appears as a left-pointing arrow on a cup or cap. So the number of left-pointing arrows in our simple closed curve is $n_{1}+n_{2}=n$. But elementary topology shows that in a (nice enough) directed, simple, closed curve the number of left-pointing critical points must always be odd (the same is true for the number of right-pointing critical points, of course). So $n$ is odd.

In the category $\operatorname{KL}(\{X\})$ we know that a simple closed curve as above is equal to $\operatorname{tr}\left(\operatorname{id}_{X}\right)$. So when $F$ is evaluated in $\mathrm{C}$ it also gives this trace. Putting everything together, we find that $f(\mathrm{C})=\operatorname{tr}\left(\mathrm{id}_{X}\right)^{n} \circ F(\mathrm{C})=\operatorname{tr}\left(\mathrm{id}_{X}\right)^{n} \circ \operatorname{tr}\left(\mathrm{id}_{X}\right)=\operatorname{tr}\left(\mathrm{id}_{X}\right)^{n+1}$. Given that $n$ is odd, this just equals the identity (using Proposition 4.20). This completes the proof for the case that the picture for $F$ has only one component.

For the multicomponent case we observe that in $\operatorname{KL}(\{X\})$ the map $F$ is the composition of the maps represented by each individual component; by what has already been argued, $F(\mathcal{C})$ is a composition of identity maps and hence equal to the identity.

Proof of Theorem 1.13 This is essentially the same as the proof of Theorem 1.6, except we use the Kelly-Laplaza category $\operatorname{KL}\left(\left\{X_{1}, \ldots, X_{n}\right\}\right)$. Note that the existence part of the theorem is obvious; the work lies in showing uniqueness of the isomorphism. For this one reduces, just as in Theorem 1.6, to the case of a composite $f$ that starts and ends with $S$ and is of the type specified in the statement of the theorem.

Such a composite $f$ is then replaced by a corresponding formal composite $F$ in $\operatorname{KL}\left(\left\{X_{1}, \ldots, X_{n}\right\}\right)$. The picture for $F$ is a collection of simple, closed curves in the plane, each labelled by one of the $X_{i}$ 's, which are allowed to intersect each other in double points. The Kelly-Laplaza theorem identifies $F$ with the composite of the maps whose pictures correspond to each closed curve. In this way one reduces to the one-variable case handled by Theorem 1.6, to conclude that $f$ must be the identity.

\subsection{Coherence with self-twists}

Proof of Theorem 1.10 The proof proceeds along the same lines as Theorem 1.6. One immediately reduces to the case $w_{1}=w_{2}=S, g=\mathrm{id}$, and where the parity of $f$ is even. Just as before, we replace $f$ by a corresponding formal composite $F$ in the Kelly-Laplaza category $\operatorname{KL}(\{X\})$. We have

$$
f(\mathcal{C})=\operatorname{tr}\left(\operatorname{id}_{X}\right)^{n} \circ F(\mathcal{C}),
$$

where the integer $n$ is the same as in the proof of Theorem 1.6. 
The picture corresponding to $F$ is no longer a union of simple curves as it was in the proof of Theorem 1.6. Rather, it is a union of oriented, closed curves that may contain double points of self-intersection. For pedagogical purposes let us first deal with the case where the picture contains a single closed curve, for example as follows:

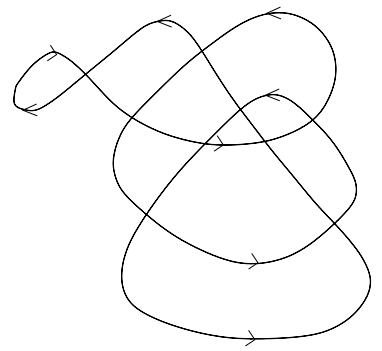

It is easy to prove that in such an oriented curve one has

(5.3) $\quad$ \# of left-pointing critical points $)+(\#$ of double points $) \equiv 1 \bmod 2$.

Indeed, let $L$ denote the number on the left of the congruence. Imagine taking a closed loop of string - an unknot - and laying it on top of the plane containing our oriented curve, in such a way that the string exactly covers the curve. This gives us an oriented knot diagram which is similar to our original picture but in which every double-point has been changed to an over- or under-crossing. One readily checks that the parity of the number $L$ is unchanged under the Reidemeister moves. Since our knot diagram is equivalent to the unknot, this says that the parity of $L$ is the same as the corresponding number for an oriented circle. But a circle clearly gives $L=1$.

The number of left-pointing critical points in the picture for $F$ is just the number $n$. Likewise, the number of double points in the picture is the parity of the formal composite $f$, which we have assumed to be even. So (5.3) tells us that $n$ is odd.

The Kelly-Laplaza theorem implies that $F(\mathcal{C})=\operatorname{tr}\left(\operatorname{id}_{X}\right)$. So $f(\mathcal{C})=\operatorname{tr}\left(\operatorname{id}_{X}\right)^{n+1}$. Since $n+1$ is even $\operatorname{tr}\left(\mathrm{id}_{X}\right)^{n+1}=\mathrm{id}_{S}$ by Proposition 4.20, and this completes the proof in the present case (where the picture for $F$ contains one closed curve).

For the general case we have $F(\mathcal{C})=\operatorname{tr}\left(\mathrm{id}_{X}\right)^{e}$ where $e$ is the number of closed curves in the picture. The analog of (5.3) - whose proof is the same as before - becomes

$$
\text { (\# of left-pointing critical points) }+(\# \text { of double points) } \equiv e \bmod 2 \text {. }
$$

We then obtain $f(\mathcal{C})=\operatorname{tr}\left(\operatorname{id}_{X}\right)^{n+e}$, and (5.4) yields that $n+e$ is even. So again we have $f(\mathcal{C})=\operatorname{id}_{S}$, as desired. 
Proof of Theorem 1.14 This is a straightforward generalization of the proof of Theorem 1.10, in the same way that Theorem 1.13 generalized Theorem 1.6. The main point is that a map from $S$ to $S$ in $\operatorname{KL}\left(\left\{X_{1}, \ldots, X_{n}\right\}\right)$ is represented by a collection of closed curves, each of which is labelled by one of the $X_{i}$ 's. The Kelly-Laplaza theorem identifies such a map with the composite of the maps obtained by considering each closed curve separately. The $i$-parity of our formal composite represents the number of double points in the curves labelled by $X_{i}$. The hypothesis that each of these parities is even guarantees, just as in the proof of Theorem 1.13, that the specified map in $\operatorname{KL}\left(\left\{X_{1}, \ldots, X_{n}\right\}\right)$ is the identity.

\section{The main applications: $\mathbb{Z}^{n}$-graded rings of maps}

Assume that $(\mathcal{C}, \otimes, S)$ is an additive category with a symmetric monoidal structure, where the tensor product is an additive functor in each variable. In this section we investigate $\mathbb{Z}^{n}$-graded groups of maps in $\mathcal{C}$.

Suppose $X_{1}, \ldots, X_{n} \in \mathcal{C}$ are invertible objects, and let $\left(X_{i}^{*}, \eta_{i}\right)$ be a specific choice of inverse for $X_{i}$. Recall the definition of $\underline{X}^{a}$ for every $a \in \mathbb{Z}^{n}$, from Section 1 . For every $a, b \in \mathbb{Z}^{n}$ there is a canonical isomorphism

$$
\phi_{a, b}: \underline{X}^{a} \otimes \underline{X}^{b} \cong \underline{X}^{a+b}
$$

specified by Theorem 1.13. The uniqueness part of that proposition guarantees that the pentagonal diagrams (2.1) all commute, and that for $a, b \in \mathbb{N}^{n}$ these $\phi_{a, b}$ 's coincide with the ones defined in Section 2.

For any $W \in \mathcal{C}$ define $\pi_{*}(W)$ to be the $\mathbb{Z}^{n}$-graded abelian group

$$
\pi_{*}(W)=\bigoplus_{a \in \mathbb{Z}^{n}} \mathcal{C}\left(\underline{X}^{a}, W\right) .
$$

Suppose that $U, V$, and $W$ are objects and that there is a pairing $U \otimes V \rightarrow W$. The maps $\phi_{a, b}$ allow us to define a $\mathbb{Z}^{n}$-graded pairing $\pi_{*}(U) \otimes \pi_{*}(V) \rightarrow \pi_{*}(W)$ as follows. Suppose $f: \underline{X}^{a} \rightarrow U$ and $g: \underline{X}^{b} \rightarrow V$. Define the product $f \cdot g$ to be the composite

$$
\underline{X}^{a+b} \stackrel{\phi_{a, b}^{-1}}{\longrightarrow} \underline{X}^{a} \otimes \underline{X}^{b} \stackrel{f \otimes g}{\longrightarrow} U \otimes V \longrightarrow W .
$$

6.1 Proposition Let $U$ be a monoid with respect to $\otimes$.

(a) $\pi_{*}(U)$ is a $\mathbb{Z}^{n}$-graded ring (associative and unital). 
(b) If $V$ is a left (resp. right) module over $U$ then $\pi_{*}(V)$ is a left (resp. right) module over $\pi_{*}(U)$.

(c) If $U$ is a commutative monoid then $\pi_{0}(U)$ is central in $\pi_{*}(U)$.

Proof The proofs of (a) and (b) are the same: distributivity is automatic, and associativity follows from the commutativity of the diagram (2.1) involving the $\phi_{a, b}$ 's. The unit conditions follow as in the proof of Proposition 2.3.

For (c), let $f: \underline{X}^{a} \rightarrow U$ and $g: S \rightarrow U$. The following diagram is commutative:

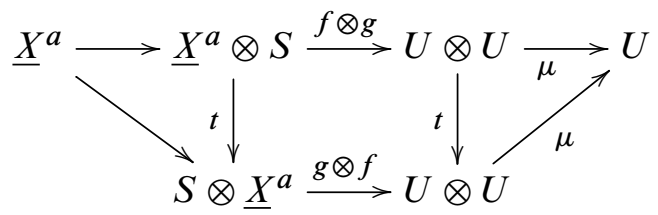

The composite across the top is $f \cdot g$, and the composite across the bottom is $g \cdot f$. Commutativity of the diagram shows these are equal.

\subsection{Representation of elements in $\pi_{*}(S)$ by maps in $\mathcal{C}$}

Let $w_{1}$ and $w_{2}$ be two tensor words in the symbols $X_{i}^{ \pm 1}$, and suppose that $f: w_{1} \rightarrow w_{2}$ is a map. Theorem 1.13 gives canonical isomorphisms $\underline{X}^{a} \rightarrow w_{1}$ and $\underline{X}^{b} \rightarrow w_{2}$ for unique $a, b \in \mathbb{Z}^{n}$. From now on we will denote all canonical isomorphisms provided by Theorem 1.13 by $\phi$. (A consequence of this is that a canonical map and its inverse which is also canonical - are sometimes both denoted by $\phi$; in practice this does not lead to much confusion, though.) Let $\langle f\rangle$ denote the composite

$$
\underline{X}^{a} \stackrel{\phi}{\rightarrow} w_{1} \stackrel{f}{\rightarrow} w_{2} \stackrel{\phi}{\rightarrow} \underline{X}^{b} .
$$

There are two evident ways to obtain an element of $\pi_{*}(S)$ from $\langle f\rangle$. Let $[f]_{r} \in \pi_{a-b}(S)$ be the composite

$$
\underline{X}^{a-b} \stackrel{\phi}{\rightarrow} \underline{X}^{-b} \otimes \underline{X}^{a} \stackrel{\text { id } \otimes\langle f\rangle}{\longrightarrow} \underline{X}^{-b} \otimes \underline{X}^{b} \stackrel{\phi}{\rightarrow} S
$$

and let $[f]_{l} \in \pi_{a-b}(S)$ be the composite

$$
\underline{X}^{a-b} \stackrel{\phi}{\rightarrow} \underline{X}^{a} \otimes \underline{X}^{-b} \stackrel{\langle f\rangle \otimes \mathrm{id}}{\longrightarrow} \underline{X}^{b} \otimes \underline{X}^{-b} \stackrel{\phi}{\rightarrow} S .
$$

In general one must be careful, as $[f]_{r}$ and $[f]_{l}$ need not be the same element. We will give a precise formula for relating them in Proposition 6.11 below, but it will take some work to build up to this. We start with some simple observations. 
6.3 Proposition Let $w_{1}, w_{2}$ and $w_{3}$ be three tensor words that are formally isomorphic to $\underline{X}^{a}, \underline{X}^{b}$ and $\underline{X}^{c}$, respectively. Let $f: w_{1} \rightarrow w_{2}, g: w_{2} \rightarrow w_{3}$. Then:

(a) $\left[\mathrm{id}_{w_{3}} \otimes f\right]_{r}=[f]_{r}$ and $\left[f \otimes \mathrm{id}_{w_{3}}\right]_{l}=[f]_{l}$

(b) $[g f]_{r}=[g]_{r} \cdot[f]_{r}$

Proof For part (a) first note that $\left\langle\mathrm{id}_{w_{3}} \otimes f\right\rangle=\mathrm{id}_{c} \otimes\langle f\rangle$, by an easy argument. Next use the following diagram, where we have suppressed some tensor signs for typographical reasons:

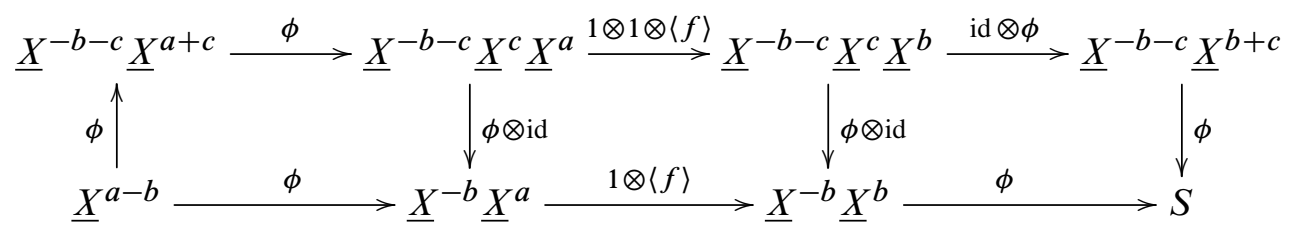

The three squares are readily checked to commute; in the case of the outer ones this is by Theorem 1.13. The composite from $\underline{X}^{a-b}$ to $S$ across the 'top' of the diagram is $\left[\operatorname{id}_{w_{3}} \otimes f\right]_{r}$, and the composite across the bottom is $[f]_{r}$. The argument showing $\left[f \otimes \mathrm{id}_{w_{3}}\right]_{l}=[f]_{l}$ is entirely similar.

For (b) we first examine the commutative diagram

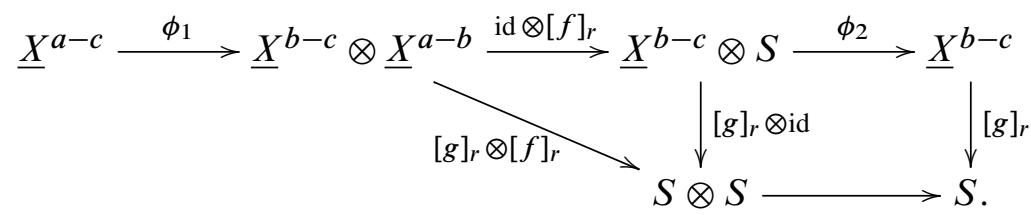

Let $H=\phi_{2} \circ\left(\operatorname{id} \otimes[f]_{r}\right) \circ \phi_{1}$. The composite across the 'bottom' of the diagram is $[g]_{r} \cdot[f]_{r}$, so we have $[g]_{r} \cdot[f]_{r}=[g]_{r} \circ H$.

Next consider the following diagram:

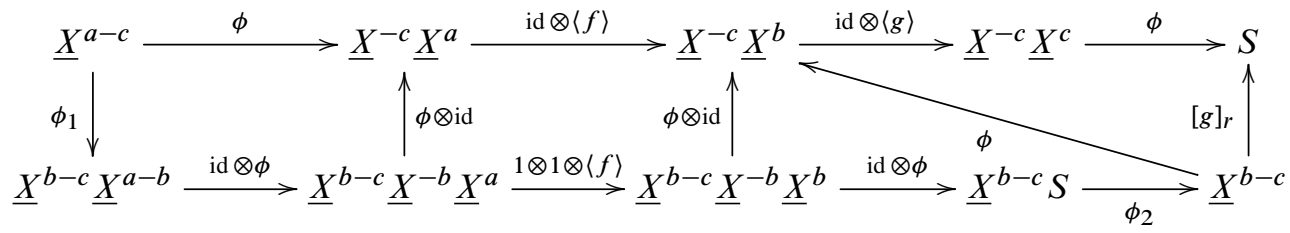

All of the regions of the diagram commute, in two cases by Theorem 1.13. The composite across the top row is $[g f]_{r}$. The composite across the bottom edge from $\underline{X}^{a-c}$ to $\underline{X}^{b-c}$ is the map $H$. So the diagram shows that $[g f]_{r}=[g]_{r} \circ H$, and the latter equals $[g]_{r} \cdot[f]_{r}$ by the preceding paragraph. 
6.4 Remark It is informative to check that the argument for part (b) of Proposition 6.3 does not dualize to prove $[g f]_{l}=[g]_{l} \cdot[f]_{l}$. The reason comes down to the fact that the formula $g \otimes f=(g \otimes \mathrm{id}) \circ(\mathrm{id} \otimes f)$ has the identity tensored on the left side of the $f$. The dual argument shows $[g f]_{l}=[f]_{l} \cdot[g]_{l}$, although we will not need this fact.

Our next task is to focus on the case where $f: w_{1} \rightarrow w_{2}$ and $w_{1} \cong w_{2} \cong \underline{X}^{a}$. Note that in this case $\langle f\rangle$ is a map $\underline{X}^{a} \rightarrow \underline{X}^{a}$ and so we also have the invariants $D(\langle f\rangle)$ and $\operatorname{tr}(\langle f\rangle)$, which like $[f]_{l}$ and $[f]_{r}$ are elements of $\pi_{0}(S)$. The following result gives the relation between all of these constructions.

6.5 Proposition Let $w_{1}$ and $w_{2}$ be two words that are formally isomorphic to $\underline{X}^{a}$, for $a \in \mathbb{Z}^{n}$. Let $f: w_{1} \rightarrow w_{2}$ be a map. Then:

(a) $[f]_{r}=[f]_{l}=D(\langle f\rangle)$ and $\operatorname{tr}(\langle f\rangle)=D(f) \cdot \operatorname{tr}\left(\operatorname{id}_{\underline{X}^{a}}\right)$.

(b) $\left[\mathrm{id}_{c} \otimes f\right]_{r}=\left[f \otimes \mathrm{id}_{c}\right]_{r}=[f]_{r}$ for any $c \in \mathbb{Z}^{n}$.

(c) For a canonical isomorphism $\phi: w_{1} \rightarrow w_{2}$ (as provided by Theorem 1.13) one has $[\phi]_{r}=\mathrm{id}_{S}$.

Before giving the proof let us introduce one more important definition. For $a, b \in \mathbb{Z}^{n}$ we have the twist map $t_{a, b}: \underline{X}^{a} \otimes \underline{X}^{b} \rightarrow \underline{X}^{b} \otimes \underline{X}^{a}$. We write $T_{a, b}=\left\langle t_{a, b}\right\rangle$, which is a map $\underline{X}^{a+b} \rightarrow \underline{X}^{a+b}$. It is easy to check that $T_{a, b} \circ T_{b, a}=\mathrm{id}$. Note that $T_{a,-a}$ is a map $S \rightarrow S$.

Proof of Proposition 6.5 For (a) we consider the following diagram:

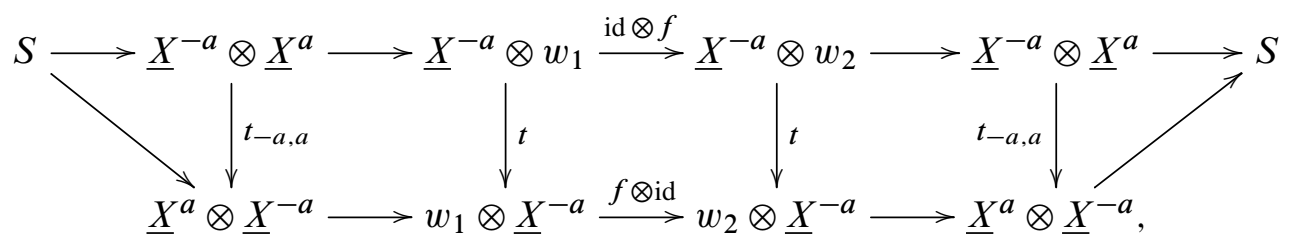

where all unlabelled maps are canonical isomorphisms (ie, they should be labelled with $\phi$ ). The three squares are commutative, but the triangles on the two ends are not; the automorphism of $S$ obtained by moving around one of these triangles is either $T_{a,-a}$ or $T_{-a, a}$, depending on which direction the composite is taken. The composite across the top of the diagram is $[f]_{r}$ and the composite across the bottom is $[f]_{l}$. The diagram thus yields the formula

$$
[f]_{r}=T_{a,-a} \circ[f]_{l} \circ T_{-a, a} .
$$


But this formula takes place in the monoid $\operatorname{End}(S)$, which by Proposition 4.7 is commutative. So we obtain $[f]_{r}=[f]_{l} \circ T_{a,-a} \circ T_{-a, a}=[f]_{l} \circ \mathrm{id}_{S}=[f]_{l}$.

Next consider the following diagram:

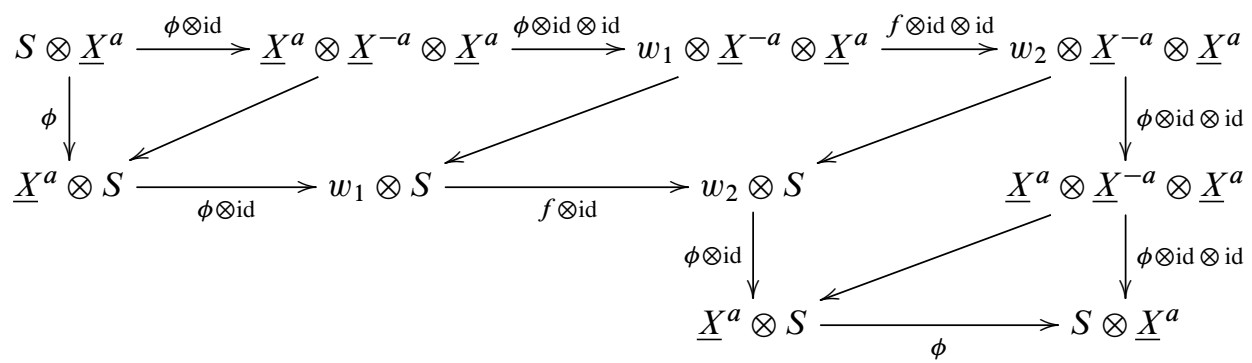

The diagonal maps are all equal to the identity on the left tensor factor and the canonical isomorphism $\phi: \underline{X}^{a} \otimes \underline{X}^{-a} \rightarrow S$ on the other two factors. All of the 'squares' obviously commute in the diagram, and the triangles on the two ends commute by Theorem 1.13 since all the maps are canonical isomorphisms. The composition across the 'top' of the diagram equals $[f]_{l} \otimes \operatorname{id}_{\underline{X}^{a}}$. Condensing the diagram to its outer rim yields the commutative square

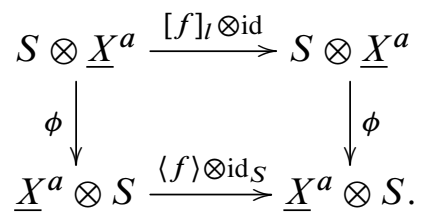

By Lemma 4.8(a) the top and bottom maps have the same $D$-invariant, and the $D$ invariant of the bottom map is also that of $\langle f\rangle$ (using the unital isomorphism). Finally, $D\left([f]_{l} \otimes \mathrm{id}\right)=D\left([f]_{l}\right)=[f]_{l}$ by Lemma 4.8(b). This ends the proof of (a).

Part (b) follows immediately from (a) and Proposition 6.3(a). Part (c) is a consequence of coherence: the composite

$$
\underline{X}^{a} \stackrel{\phi_{1}}{\longrightarrow} w_{1} \stackrel{\phi}{\rightarrow} w_{2} \stackrel{\phi_{2}}{\longrightarrow} \underline{X}^{a}
$$

is a canonical map and must therefore equal the identity by Theorem 1.13 . Hence, $\langle\phi\rangle=\operatorname{id}_{\underline{X}^{a}}$ and $[\phi]_{r}=D\left(\operatorname{id}_{\underline{X}^{a}}\right)=\mathrm{id}_{S}$.

The elements $T_{a, b} \in \operatorname{Aut}(S)$ are, of course, ubiquitous in calculations. We define

$$
\tau_{a, b}=\left[t_{a, b}\right]_{l}=\left[t_{a, b}\right]_{r}=D\left(T_{a, b}\right) \in \pi_{0}(S),
$$

where the second two equalities are by Proposition 6.5. Recall that we have the basic commuters $\tau_{i}=\operatorname{tr}\left(\operatorname{id}_{X_{i}}\right) \in \pi_{0}(S)$ and these satisfy $\tau_{i}^{2}=1$ by Proposition 4.20 . Recall 
as well that $\tau_{i}=D\left(t_{X_{i}}, X_{i}\right)$ by Proposition 4.24. If $e_{1}, \ldots, e_{n}$ is the standard basis for $\mathbb{Z}^{n}$ then this just says that $\tau_{i}=\tau_{e_{i}, e_{i}}$. Let us also point out that if $i \neq j$ then $\tau_{e_{i}, e_{j}}=\mathrm{id}_{S} ;$ in fact $T_{e_{i}, e_{j}}$ is the composite

$$
\underline{X}^{e_{i}+e_{j}} \stackrel{\phi}{\rightarrow} X_{i} \otimes X_{j} \stackrel{t_{X_{i}, X_{j}}}{\longrightarrow} X_{j} \otimes X_{i} \stackrel{\phi}{\rightarrow} \underline{X}^{e_{i}+e_{j}}
$$

and this equals the identity map either by Theorem 1.14 or by just looking at the definitions. Quite generally, we can express all of the elements $\tau_{a, b}$ in terms of the basic commuters.

6.6 Proposition For all $a, b \in \mathbb{Z}^{n}$ one has $\tau_{a, b}=\tau_{1}^{\left(a_{1} b_{1}\right)} \cdots \tau_{n}^{\left(a_{n} b_{n}\right)}$.

Proof Recall that $T_{a, b}$ is the composite $\underline{X}^{a+b} \stackrel{\phi}{\rightarrow} \underline{X}^{a} \otimes \underline{X}^{b} \stackrel{t_{a, b}}{\rightarrow} \underline{X}^{b} \otimes \underline{X}^{a} \stackrel{\phi}{\rightarrow} \underline{X}^{a+b}$. Observe that we can also obtain this map as a long composite

$$
\underline{X}^{a+b} \rightarrow w_{1} \rightarrow w_{2} \rightarrow \cdots \rightarrow w_{N} \rightarrow \underline{X}^{a+b},
$$

where each $w_{k}$ is a tensor word in the $X_{i}^{ \pm 1}$ 's and each map is one of the following:

(1) a canonical isomorphism $\phi$ (as provided by Theorem 1.13)

(2) a tensor product of $t_{X_{i}, X_{i}}$ with identity maps

(3) a tensor product of $t_{X_{i}, X_{i}^{-1}}$ with identity maps

(4) a tensor product of $t_{X_{i}^{-1}, X_{i}^{-1}}$ with identity maps

In the 'standard' way to obtain such a composite the number of transpositions of types (2)-(4) will be $\left|a_{i} b_{i}\right|$, for any chosen value of $i$. Let $f: S \rightarrow S$ be the map $\prod_{i} \tau_{i}^{\left(a_{i} b_{i}\right)}$, noting that only the parity of $a_{i} b_{i}$ matters in the exponent since $\tau_{i}^{2}=\mathrm{id}_{S}$. Consider the composite

$$
\underline{X}^{a} \stackrel{\phi}{\rightarrow} S \otimes \underline{X}^{a} \stackrel{f \otimes \mathrm{id}}{\longrightarrow} S \otimes \underline{X}^{a} \stackrel{\phi}{\rightarrow} \underline{X}^{a} .
$$

It follows from Theorem 1.14 that the composites in (6.7) and (6.8) are the same, because by construction they have the same $i$-parity for every $i$. Consequently, the $D-$ invariant of the two composites is the same. But the $D$-invariant of (6.8) is manifestly equal to the map $f$. We have thus proven that $f=D\left(T_{a, b}\right)=\tau_{a, b}$.

6.9 Remark As a consequence of Proposition 6.6 and the fact that $\tau_{i}^{2}=1$ note that we have $\tau_{a, b} \tau_{a, c}=\tau_{a, b+c}=\tau_{a, b-c}$. Likewise, $\tau_{a, b}=\tau_{-a, b}=\tau_{b, a}$. Identities such as these will often be used. 
Before proceeding further we need a lemma, which is easy but worth recording.

6.10 Lemma Consider composable maps

$$
\underline{X}^{a} \stackrel{f}{\rightarrow} \underline{X}^{a} \stackrel{g}{\rightarrow} S \stackrel{h}{\rightarrow} S .
$$

Then as elements of $\pi_{*}(S)$ one has $h g=h \cdot g=g \cdot h$ and $g f=g \cdot D(f)$. More generally, we can write $h g f=h \cdot g \cdot D(f)=g \cdot h \cdot D(f)$.

Proof We have already seen that $h \cdot g=g \cdot h$, in Proposition 2.3. The identification of these with the composite $h g$ is easy. For the second identity we have

$$
g \circ f=g \circ\left(\operatorname{id}_{X} \hat{\otimes} D(f)\right)=g \hat{\otimes} D(f)=g \cdot D(f),
$$

where the second equality is from Lemma 4.2 and the third equality follows from the definitions. Finally, the identity for $h g f$ is a consequence of the previous identities.

Now we can move on to the study of $[f]_{r}$ and $[f]_{l}$ for general maps $f$.

6.11 Proposition Let $w_{1}$ and $w_{2}$ be two tensor words, where $w_{1}$ is formally isomorphic to $\underline{X}^{a}$ and $w_{2}$ is formally isomorphic to $\underline{X}^{b}$. Let $f: w_{1} \rightarrow w_{2}$, and let $c \in \mathbb{Z}^{n}$. Write $\mathrm{id}_{c}$ for $\mathrm{id}_{\underline{X}^{c}}$. Then:

(a) $[f]_{r}=[f]_{l} \cdot \tau_{b, a-b}$

(b) $\left[\operatorname{id}_{c} \otimes f\right]_{r}=[f]_{r}$

(c) $\left[f \otimes \mathrm{id}_{c}\right]_{r}=[f]_{r} \cdot \tau_{a-b, c}$

(d) $\left[f \otimes \operatorname{id}_{c}\right]_{l}=[f]_{l}$

(e) $\left[\mathrm{id}_{c} \otimes f\right]_{l}=[f]_{l} \cdot \tau_{a-b, c}$

(f) if $g: w_{2} \rightarrow w_{3}$, where $w_{3} \cong \underline{X}^{c}$, then $[g f]_{r}=[g]_{r} \cdot[f]_{r}$ and likewise $[g f]_{l}=$ $[g]_{l} \cdot[f]_{l} \cdot \tau_{a-b, c-d}$

(g) let $g: w_{1}^{\prime} \rightarrow w_{2}^{\prime}$ where $w_{1}^{\prime} \cong \underline{X}^{c}$ and $w_{2}^{\prime} \cong \underline{X}^{d}$, then

$$
\begin{aligned}
{[f \otimes g]_{r} } & =[f]_{r} \cdot[g]_{r} \cdot \tau_{a-b, d}=[g]_{r} \cdot[f]_{r} \cdot \tau_{a-b, c} \\
{[f \otimes g]_{l} } & =[f]_{l} \cdot[g]_{l} \cdot \tau_{b, c-d}=[g]_{l} \cdot[f]_{l} \cdot \tau_{a, c-d}
\end{aligned}
$$

Proof of Proposition 6.11 Note first that parts (b), (d), and the first part of (f) were already proven in Proposition 6.3; they are only restated here for ease of reference. Note also that parts (c), (e), and the second part of (f) are formal consequences of the aforementioned results, using (a). So most everything follows from (a). 
To prove (a) we consider the usual diagram:

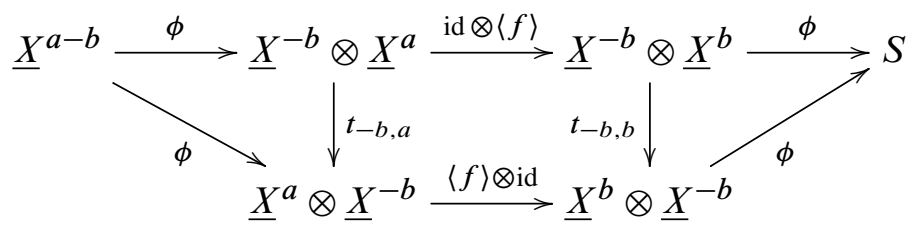

The square commutes but the triangles do not; the composite across the top is $[f]_{r}$ and the composite across the bottom is $[f]_{l}$. The diagram yields the formula

$$
[f]_{r}=T_{b,-b} \circ[f]_{l} \circ T_{-b, a} .
$$

From Lemma 6.10 we get that in $\pi_{*}(S)$ one has the formula

$$
[f]_{r}=[f]_{l} \cdot T_{b,-b} \cdot \tau_{-b, a}=[f]_{l} \cdot \tau_{b,-b} \cdot \tau_{-b, a}=[f]_{l} \cdot \tau_{a-b, b}
$$

(using Remark 6.9 for the final equality).

Finally, in $(\mathrm{g})$ we simply use that $f \otimes g=\left(\mathrm{id}_{w_{2}} \otimes g\right) \circ\left(f \otimes \mathrm{id}_{w_{1}^{\prime}}\right)=\left(f \otimes \mathrm{id}_{w_{2}^{\prime}}\right) \circ$ $\left(\mathrm{id}_{w_{1}} \otimes g\right)$. The desired formulas follow from the combined application of the previous parts.

\subsection{Skew-commutativity}

Skew-commutativity for $\pi_{*}(S)$ follows immediately from the various formulas in Proposition $6.11(\mathrm{~g})$. We give a slightly more general version here.

6.13 Proposition Let $W$ be an object in $\mathcal{C}$, let $f: \underline{X}^{a} \rightarrow S$ and $g: \underline{X}^{b} \rightarrow W$. Then under the left and right actions of $\pi_{*}(S)$ on $\pi_{*}(W)$ we have

$$
f \cdot g=g \cdot f \cdot \tau_{a, b}=g \cdot f \cdot \tau_{1}^{\left(a_{1} b_{1}\right)} \cdots \tau_{n}^{\left(a_{n} b_{n}\right)} .
$$

Proof Consider the diagram

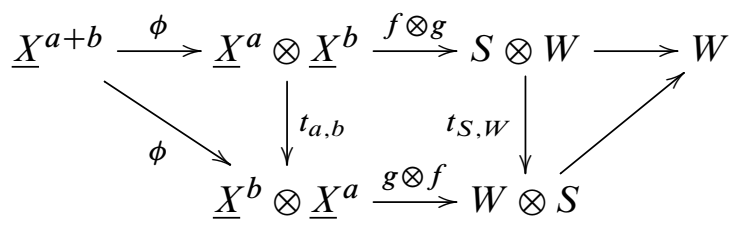

and note that all regions commute except the leftmost triangle. The composite across the top is $f \cdot g$, and the composite across the bottom is $g \cdot f$. The diagram yields the identity $f \cdot g=(g \cdot f) \circ A$, where $A$ is the appropriate self-map of $\underline{X}^{a+b}$ coming from the left triangle. By Lemma 6.10 we obtain $f \cdot g=g \cdot f \cdot D(A)$ in $\pi_{*}(W)$, and 
we know that $D(A)=\left[t_{a, b}\right]_{r}=\tau_{a, b}$. The identification of $\tau_{a, b}$ as $\prod_{i} \tau_{i}^{\left(a_{i} b_{i}\right)}$ is from Proposition 6.6.

6.14 Remark There are other settings in which one can prove similar skew-commutativity results. For example, if $W$ is a commutative monoid in $\mathcal{C}$ (with respect to $\otimes$ ) then $\pi_{*}(W)$ has the same skew-commutativity law as $\pi_{*}(S)$, where now the $\tau_{i}$ 's are regarded as elements of $\pi_{*}(W)$ via the unit map $S \rightarrow W$. If $Z$ is a bimodule over $W$ then there is a corresponding skew-commutativity result in that setting. All of the proofs are the same as for Proposition 6.13 above, so we leave these to the reader.

\section{More general grading schema}

Let $(\mathrm{C}, \otimes, S)$ be an additive category with a symmetric monoidal structure that is additive in each variable. Let $A$ be a finitely-generated abelian group, and fix a homomorphism $h: A \rightarrow \operatorname{Pic}(\mathcal{C})$. For each $a \in A$ let $X_{a}$ be a chosen object in the isomorphism class $h(a)$; assume $X_{0}=S$. For $W$ in $\mathcal{C}$ define $\pi_{*}^{A}(W)$ to be the $A$-graded abelian group $a \mapsto \mathcal{C}\left(X_{a}, W\right)$. To obtain a product on $\pi_{*}^{A}(S)$ one can start by choosing isomorphisms

$$
\sigma_{a, b}: X_{a+b} \rightarrow X_{a} \otimes X_{b}
$$

for each $a, b \in A$. If $f: X_{a} \rightarrow S$ and $g: X_{b} \rightarrow S$ then we define the product $f \cdot g$ to be the composite

$$
X_{a+b} \stackrel{\sigma_{a, b}}{\longrightarrow} X_{a} \otimes X_{b} \stackrel{f \otimes g}{\longrightarrow} S \otimes S \cong S .
$$

This clearly defines a distributive product on $\pi_{*}^{A}(S)$. The questions that arise are:

(1) Is it possible to choose the $\sigma_{a, b}$ isomorphisms so that the product on $\pi_{*}^{A}(S)$ is associative and unital?

(2) If there are multiple ways to accomplish (1), do they give rise to isomorphic rings? That is, is the ring structure on $\pi_{*}^{A}(S)$ in some sense canonical?

Note that in Section 6 we proved that the answer to (1) is yes in the case when $A$ is free. The construction depended on choosing a free basis $e_{1}, \ldots, e_{n}$ for $A$ and then fixing a specific choice of isomorphism $\eta_{i}: S \rightarrow X_{-e_{i}} \otimes X_{e_{i}}$ for each $i$; so the construction was certainly not canonical.

We will see below that the answer to (1) is yes in general, but the answer to (2) is no. In fact, the set of isomorphism classes of ring structures obtained in this way is parameterized by the cohomology group $H^{2}(A ; \operatorname{Aut}(S))$. Much of the material behind 
this story seems to be standard, but we were unable to find an adequate reference (the introduction to the paper [2] by Cegarra and Khmaladze gives a partial survey, though). I am grateful to Victor Ostrik and Vadim Vologodsky for conversations about the results in this section.

Let us call the collection $\left(\sigma_{a, b}\right)_{a, b \in A}$ an $A$-trivialization of $\mathcal{C}$ with respect to $X$ if it satisfies two properties:

(1) For every $a \in A$ the isomorphisms $\sigma_{a, 0}$ and $\sigma_{0, a}$ coincide with the unital isomorphisms in $\mathcal{C}$.

(2) For every $a, b, c \in A$ the following pentagon commutes:

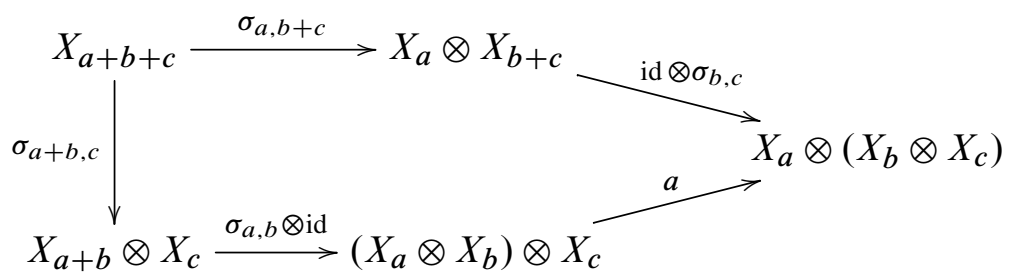

Under conditions (1) and (2) the induced product on $\pi_{*}^{A}(S)$ is both associative and unital; we will call this the standard ring structure on $\pi_{*}^{A}(S)$ associated to $\sigma$. Note that there possibly exist ring structures on $\pi_{*}^{A}(S)$ which are not standard, ie, which do not arise from an $A$-trivialization. Such structures are not part of the theory we develop here.

7.1 Remark Let $\underline{A}$ be the category having only identity maps and whose objects are the elements of $A$; equip $\underline{A}$ with the evident symmetric monoidal structure coming from the group law in $A$. Then an $A$-trivialization of $\mathcal{C}$ with respect to $X$ is simply the extra structure needed to make $X$ into a strong monoidal functor that is strictly unital.

Given two $A$-trivializations $\sigma$ and $\sigma^{\prime}$ we get two ring structures $\pi_{*}^{A}(S)_{\sigma}$ and $\pi_{*}^{A}(S)_{\sigma^{\prime}}$. Are the two standard rings obtained in this way isomorphic? The question is not easy to answer when stated so broadly, but we can refine it somewhat. The evident way to construct a map $\pi_{*}^{A}(S)_{\sigma} \rightarrow \pi_{*}^{A}(S)_{\sigma^{\prime}}$ would be to send each $f: X_{a} \rightarrow S$ to $f \cdot u(a)$ for some chosen $u(a) \in \operatorname{Aut}(S)$ that is independent of $f$. [Note that it does not matter which product we use for $f \cdot u(a)$, since both the $\sigma$-product and the $\sigma^{\prime}$-product will give the same answer if one of the factors lies in $\pi_{0}^{A}(S)$, by condition (1).] Let us say that a standard isomorphism between standard ring structures is one that is of this form; note that it is determined by a chosen map of sets $u: A \rightarrow \operatorname{Aut}(S)$.

Here is the main goal of this section. 
7.2 Proposition Suppose $(\mathcal{C}, \otimes, S), h: A \rightarrow \operatorname{Pic}(\mathcal{C})$, and $X: A \rightarrow \mathrm{ob}(\mathcal{C})$ are as in the beginning of this section.

(a) There exists an $A$-trivialization of $\mathcal{C}$ with respect to $X$, and therefore a resulting standard ring structure on $\pi_{*}^{A}(S)$.

(b) The set of all $A$-trivializations as in (a) is in bijective correspondence with $Z^{2}(A ; \operatorname{Aut}(S))_{\text {norm }}$, the normalized 2-cocycles for the bar complex of $A$.

(c) The set of different possible standard ring structures on $\pi_{*}^{A}(S)$, up to standard isomorphism, is in bijective correspondence with $H^{2}(A ; \operatorname{Aut}(S))$.

Note that we essentially already encountered this in the case where $A$ was $\mathbb{Z}^{n}$. In that case $H^{2}(A ; \operatorname{Aut}(S)) \cong \operatorname{Aut}(S)^{n}$ (noncanonically), and one only obtains a ring structure after fixing a basis for $A$ together with $n$ elements of $\operatorname{Aut}(S)$, as we found in our earlier treatment. The overall lesson is that grading morphism sets by invertible objects is a bit dicey when it comes to product structures; the rings obtained are typically neither unique nor canonical.

7.3 Remark One can also ask about the graded commutativity properties of $\pi_{*}^{A}(S)$. It is easy to prove that if $f \in \pi_{a}^{A}(S)$ and $g \in \pi_{b}^{A}(S)$, then $f g=g f \cdot \theta_{a, b}$, where $\theta_{a, b}=D\left(\sigma_{b, a}^{-1} \circ t_{a, b} \circ \sigma_{a, b}\right) \in \operatorname{Aut}(S)$. We have not explored the properties of $\theta: A^{2} \rightarrow \operatorname{Aut}(S)$, mostly due to a lack of application. Our analysis in the free case (Proposition 6.6) suggests this might be a nice exercise.

We will prove Proposition 7.2 by analyzing a very specific class of monoidal categories, and then reducing to that case.

\subsection{Monoidal categories of type $(A, N)$}

Fix abelian groups $A$ and $N$. Let $\mathcal{C}=\mathcal{C}[A, N]$ be the category with object set $A$, where there are no maps between distinct objects, and where the set of self-maps of each object is equal to $N$. Define a bifunctor $\otimes: \mathcal{C} \times \mathcal{C} \rightarrow \mathcal{C}$ whose behavior on objects is given by the sum in $A$, and whose behavior on morphisms is given by the sum in $N$. To equip $\left(\mathcal{C}, \otimes, 0_{A}\right)$ with a monoidal structure we must specify unital isomorphisms $a \oplus 0 \cong a$ and $0 \oplus a \cong a$; but $a \oplus 0=a=0 \oplus a$, so we can (and will) just take the isomorphisms to be the identities.

We must also specify an associativity isomorphism $\alpha_{a, b, c}:(a \otimes b) \otimes c \rightarrow a \otimes(b \otimes c)$, for every $a, b, c \in A$. Again, since the objects $(a \otimes b) \otimes c$ and $a \otimes(b \otimes c)$ are actually equal (they both are equal to the object $a+b+c$ ) we are just specifying an element 
$\alpha_{a, b, c} \in N$. We could require this to be the identity, but we wish to not be so restrictive here. Let us call a monoidal structure on $\left(\mathcal{C}, \otimes, 0_{A}\right)$ obtained in this way an extended monoidal structure, as it is an extension of the canonical tensor functor and unital isomorphisms.

The pentagonal condition that a monoidal structure must satisfy says that $\alpha: A^{3} \rightarrow N$ is a 3 -cocycle in the usual bar complex $C^{*}(A ; N)$ for computing group cohomology. Compatibility between associativity and unital isomorphisms then requires that $\alpha_{a, b, c}=0$ if any of $a, b$, or $c$ are zero; in other words, we have a normalized cocycle. In this way we see that extended monoidal structures on $(\mathcal{C}, \otimes, 0)$ are in bijective correspondence with the group $Z^{3}(A ; N)$ norm. Even more, it is easy to see that elements of the group $H^{3}(A ; N)$ are in bijective correspondence with extended monoidal structures on $(\mathrm{C}, \otimes, 0)$ up to isomorphism. This is a standard story. For $\alpha \in Z^{3}(A ; N)_{\text {norm }}$ write $\mathrm{C}_{\alpha}=\mathrm{e}[A, N]_{\alpha}$ for the corresponding monoidal category.

Fix an element $\alpha \in Z^{3}(A ; N)$ norm. In $\bigodot_{\alpha}$ let us ask if there is an $A$-trivialization with respect to the identity map: that is, do there exist isomorphisms $\sigma_{a, b}: a \otimes b \rightarrow a+b$ satisfying the required associativity and unital conditions? Again, $\sigma_{a, b}$ is just an element of $N$ and so $\sigma \in C^{2}(A ; N)$. The unital condition is the requirement $\sigma \in C^{2}(A ; N)_{\text {norm }}$ and the associativity condition translates to $\delta \sigma=\alpha$. So the cohomology class of $\alpha$

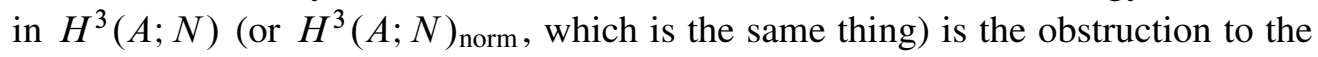
existence of the desired $\sigma$ 's.

7.5 Remark Note that the $\sigma_{a, b}$ 's are giving a (strong) monoidal structure on the identity functor $\mathrm{C}[A, N]_{0} \rightarrow \mathrm{C}[A, N]_{\alpha}$, showing that the domain and target are monoidally equivalent. This is why we call the collection $\left(\sigma_{a, b}\right)_{a, b \in A}$ a trivialization of the monoidal structure $\mathrm{e}[A, N]_{\alpha}$.

\subsection{Symmetric monoidal categories of type $(A, N)$}

There is a similar story for the existence of extended symmetric monoidal categories on $\mathcal{C}$. Here one must specify both the $\alpha_{a, b, c}$ elements and certain elements $\beta_{a, b} \in N$ giving the commutativity isomorphisms. One again finds that the set of extended structures is in bijective correspondence with the 3-cocycles in a certain complex. To describe this, let $\mathcal{E}$ be the complex

$\mathbb{Z}\left\langle A^{4}\right\rangle \oplus \mathbb{Z}\left\langle A^{3}\right\rangle_{1} \oplus \mathbb{Z}\left\langle A^{3}\right\rangle_{2} \oplus \mathbb{Z}\left\langle A^{2}\right\rangle \stackrel{d_{4}}{\longrightarrow} \mathbb{Z}\left\langle A^{3}\right\rangle \oplus \mathbb{Z}\left\langle A^{2}\right\rangle \stackrel{d_{3}}{\longrightarrow} \mathbb{Z}\left\langle A^{2}\right\rangle \stackrel{d_{2}}{\longrightarrow} \mathbb{Z}\langle A\rangle \stackrel{d_{1}}{\longrightarrow} 0$

(concentrated in homological degrees 0 through 4 ) with differentials defined on free generators by the formulas below: 


$$
\begin{gathered}
d_{1}([a])=0, \quad d_{2}([a \mid b])=[a]-[a+b]+[b] \\
d_{3}([a|b| c])=[b \mid c]-[a+b \mid c]+[a \mid b+c]-[a \mid b], \quad d_{3}([a \mid b])=[a \mid b]-[b \mid a] \\
d_{4}([a|b| c \mid d])=[b|c| d]-[a+b|c| d]+[a|b+c| d]-[a|b| c+d]+[a|b| c] \\
d_{4}\left([a|b| c]_{1}\right)=[a|b| c]-[a|c| b]+[c|a| b]-[b \mid c]+[a+b \mid c]-[a \mid c] \\
d_{4}\left([a|b| c]_{2}\right)=[a|b| c]-[b|a| c]+[b|c| a]+[a \mid b]-[a \mid b+c]+[a \mid c] \\
d_{4}([a \mid b])=[a \mid b]+[b \mid a]
\end{gathered}
$$

Let $D \subseteq \mathcal{E}$ be the "degenerate" subcomplex spanned by all symbols $\left[a_{1}|\cdots| a_{n}\right]$ in which at least one of the $a_{i}$ 's is zero, and note that this is indeed closed under the differential. A little legwork shows that extended symmetric monoidal structures on $\mathcal{C}$ correspond to normalized 3-cocycles $(\alpha, \beta) \in Z^{3}(\operatorname{Hom}(\mathcal{E}, N))$ (where 'normalized' refers to cocycles that vanish on the degenerate subcomplex).

7.7 Remark The paper [7] used a similar complex but where the $\mathbb{Z}\left\langle A^{3}\right\rangle_{2}$ term was omitted from $\mathcal{E}_{4}$ (and where the grading of the complex was shifted by 1 ). It is easy to see that omitting this term does not effect $H_{3}(\varepsilon)$ or $Z^{3}(\varepsilon ; N)$; in effect, the relations coming from this term are consequences of the ones coming from $d_{4}\left([a|b| c]_{1}\right)$ and $d_{4}([a \mid b])$, by an easy exercise. We are using the larger complex because it allows us to directly quote published results from Eilenberg and Mac Lane [5].

The complex $\mathcal{E}$ was introduced by Eilenberg and Mac Lane [4; 5]: it is the first few terms of their iterated bar construction. They prove that their complex calculates the homology of Eilenberg-Mac Lane spaces in the stable range; in particular,

$$
H_{i}(\mathcal{E}) \cong H_{n+i-1}(K(A, n))
$$

for $1 \leq i \leq 3$ and $n \geq 3$ [4, Theorem 6]. Let us write $H_{*}^{E M}(A)$ for $H_{*}(\mathcal{E})$ and $H_{E M}^{*}(A ; N)$ for $H^{*}(\operatorname{Hom}(\mathcal{E}, N))$. Eilenberg and Mac Lane calculated the following.

7.8 Proposition (Eilenberg-Mac Lane)

(a) There are natural isomorphisms

$$
H_{1}^{E M}(A) \cong A, \quad H_{2}^{E M}(A) \cong 0, \quad H_{3}^{E M}(A) \cong A / 2 A .
$$

The last isomorphism is induced by $a \mapsto[a \mid a]$.

(b) There is an isomorphism $H_{E M}^{3}(A ; N) \rightarrow \operatorname{Hom}(A / 2 A, N)=\operatorname{Hom}\left(A,{ }_{2} N\right)$ given by $(\alpha, \beta) \mapsto[x \mapsto \beta(x, x)]$.

The isomorphisms in part (a) are from [5, Theorems 20.3, 20.5, 23.1]. Note that (b) is an immediate consequence of (a), using the universal coefficient theorem. Also, note that part of the claim in (b) is that if $(\alpha, \beta)$ is a 3 -cocycle in $\operatorname{Hom}(\mathcal{E}, N)$ then 
$x \mapsto \beta(x, x)$ is linear and takes its values in ${ }_{2} N$. Neither of these claims is immediately obvious, although they follow from (a). Separate from this, however, observe that they also follow from Proposition 4.23 because $x \mapsto \beta(x, x)$ is the $\tau$-function for the symmetric monoidal category $\mathrm{C}[A, N]_{(\alpha, \beta)}$.

Observe that the bar complex $C_{*}(A)$ is contained inside $\mathcal{E}$ as a subcomplex. Let $Q$ be the quotient, so that we have the short exact sequence $0 \rightarrow C_{*}(A) \rightarrow \mathcal{E} \rightarrow Q \rightarrow 0$. Note that $Q$ has the form $\mathbb{Z}\left\langle A^{3}\right\rangle_{1} \oplus \mathbb{Z}\left\langle A^{3}\right\rangle_{2} \oplus \mathbb{Z}\left\langle A^{2}\right\rangle \rightarrow \mathbb{Z}\left\langle A^{2}\right\rangle$, concentrated in degrees 3 and 4. Applying $\operatorname{Hom}(-, N)$, the long exact sequence in cohomology then gives

$$
\cdots \leftarrow H^{3}(A ; N) \leftarrow H_{E M}^{3}(A ; N) \leftarrow H^{3}(Q ; N) \leftarrow H^{2}(A ; N) \leftarrow \cdots .
$$

The group $H^{3}(Q ; N)$ is easy to analyze: it is the collection of $\beta: A^{2} \rightarrow N$ satisfying $\beta(x, y)=-\beta(y, x)$ and $\beta(y, z)-\beta(x+y, z)+\beta(x, z)=0$ for all $x, y, z \in A$. In other words, $H^{3}(Q ; N)$ is the collection of alternating bilinear forms $A \times A \rightarrow N$; write this as $H^{3}(Q ; N) \cong \operatorname{AltBilin}(A, N)$. The map $\operatorname{AltBilin}(A, N) \rightarrow H_{E M}^{3}(A ; N)$ sends an alternating form $\beta$ to the cohomology class $[(0, \beta)]$.

The following lemma is the key calculation of this entire section.

7.10 Lemma For any abelian groups $A$ and $N$, the map $H_{E M}^{3}(A ; N) \rightarrow H^{3}(A ; N)$ is the zero map.

Proof We consider the commutative diagram

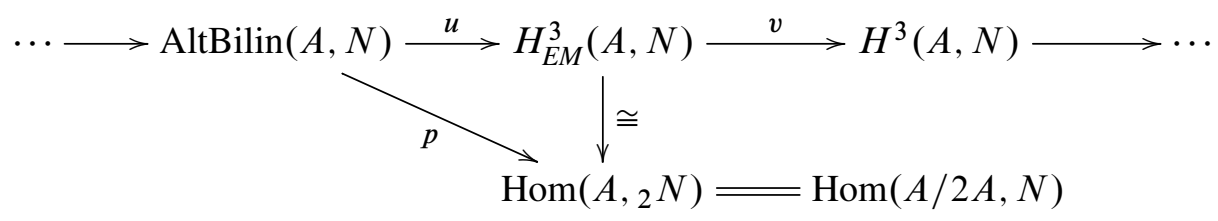

where the top row is the long exact sequence (7.9), the vertical map is the one from Proposition 7.8(b), and the map $p$ is the evident composite. Note that $p$ sends an alternating bilinear form $\theta: A \times A \rightarrow N$ to the map $a \mapsto \theta(a, a)$. But it is easy to see that $p$ is surjective. Indeed, let $\left\{e_{i}\right\}$ be an $\mathbb{F}_{2}$-basis for $A / 2 A$. If $f: A / 2 A \rightarrow N$ then define a bilinear form $b: A / 2 A \times A / 2 A \rightarrow N$ by $b\left(e_{i}, e_{j}\right)=0$ if $i \neq j$ and $b\left(e_{i}, e_{i}\right)=f\left(e_{i}\right)$. This is alternating because $2 f\left(e_{i}\right)=0$. Let $\tilde{b}$ be the composite $A \times A \rightarrow A / 2 A \times A / 2 A \stackrel{b}{\longrightarrow} N$, and note that $p(\widetilde{b})=f$.

Since $p$ is surjective it follows that $u$ is surjective, and so $v=0$. 


\subsection{Trivializations of $(A, N)$-structures}

7.12 Proposition Fix an extended symmetric monoidal structure $(\alpha, \beta)$ on $\mathrm{C}[A, N]$. Then there exists a trivialization of the monoidal structure $\mathcal{C}[A, N]_{\alpha}$, and the set of all such trivializations is in bijective correspondence with $Z^{2}(A ; N)_{\text {norm }}$.

Proof As we saw in 7.4, a trivialization is simply an element $\sigma \in C^{2}(A ; N)_{\text {norm }}$ satisfying $\delta \sigma=\alpha$. It is clear that if such a thing exists, the set of all possibilities is in bijective correspondence with $Z^{2}(A ; N)_{\text {norm }}$. To prove existence we need only show that $[\alpha]=0$ in $H^{3}(A ; N)$. But the map $H_{E M}^{3}(A ; N) \rightarrow H^{3}(A ; N)$ which sends $[(\alpha, \beta)]$ to $[\alpha]$ is the zero map by Lemma 7.10, so this finishes the proof.

\subsection{The general case}

We will prove Proposition 7.2 by reducing the construction of an $A$-trivialization to the corresponding problem for an extended symmetric monoidal category of type $(A, N)$. This uses the following lemma from [7, Chapter II, Proposition 7].

7.14 Lemma Let $(\mathcal{C}, \otimes, S)$ be a symmetric monoidal category in which every object is invertible and every map is an isomorphism. Then $\mathcal{C}$ is equivalent (as a symmetric monoidal category) to an extended symmetric monoidal category of type $(\operatorname{Pic}(\mathcal{C}), \operatorname{Aut}(S))$.

Proof First recall that $\mathcal{C}$ is equivalent to a symmetric monoidal category where the associativity and unital conditions are strict [13, Theorem XI.3.1]. So we can just assume that $\mathcal{C}$ itself has these properties.

Let $N=\operatorname{Aut}(S)$. Let $\mathcal{D}$ be the category whose objects are the elements of $\operatorname{Pic}(\mathcal{C})$, where there are no maps between distinct objects, and where every endomorphism of self-maps is equal to $N$.

For each element $a \in \operatorname{Pic}(\mathcal{C})$ choose a fixed object $X_{a}$ in $\mathcal{C}$ that belongs to this isomorphism class; when $a=[S]$ choose $X_{a}=S$. Moreover, for each $Y$ in $\mathcal{C}$ choose a fixed isomorphism $i_{Y}: Y \rightarrow X_{[Y]}$. Define a functor $F: \mathcal{C} \rightarrow \mathcal{D}$ by sending each object $Y$ to its isomorphism class in $\operatorname{Pic}(\mathcal{C})$; if $g: Y_{1} \rightarrow Y_{2}$ is a map, then let $F(g)=D\left(i_{Y_{2}} \circ g \circ i_{Y_{1}}^{-1}\right)$. One readily checks that this is indeed a functor, and that each self-map $f: Y \rightarrow Y$ is sent to its $D$-invariant $D(f) \in N$.

Likewise, define a functor $G: \mathcal{D} \rightarrow \mathcal{C}$ by sending an object $a \in \operatorname{Pic}(\mathcal{C})$ to $X_{a}$, and sending a self-map of $a$ corresponding to $n \in N$ to the unique self-map $X_{a} \rightarrow X_{a}$ that has $D$-invariant equal to $n$. It is easy to check that $F$ and $G$ give an equivalence of categories. Note that $F G=\mathrm{id}_{\mathcal{D}}$ and that $G([S])=S$. 
Use the equivalence $(F, G)$ to transplant the symmetric monoidal structure from $\mathcal{C}$ onto $\mathcal{D}$. For example, define the monoidal product on $\mathcal{D}$ by

$$
d_{1} \otimes d_{2}=F\left(G d_{1} \otimes G d_{2}\right),
$$

and likewise for the associativity, unital, and commutativity isomorphisms. It is routine to check that the unit in $\mathcal{D}$ is strict, because this was assumed to be the case for $\mathcal{C}$ and $G([S])=S$; in contrast, the associativity isomorphisms need not be strict. But one readily verifies that this gives an extended symmetric monoidal structure on $\mathcal{D}$, which is equivalent to $(\mathcal{C}, \otimes, S)$ by construction.

Proof of Proposition 7.2 We begin by replacing $\mathcal{C}$ by the subcategory $\mathcal{C}^{\text {inv }}$ of invertible objects and isomorphisms: the question of whether or not there exists an $A$-trivialization of $X$ is the same for $\mathcal{C}$ and $\mathcal{C}^{\text {inv }}$. Next use Lemma 7.14 to replace $\mathcal{C}^{\text {inv }}$ by an extended symmetric monoidal category of type $(A, N)$, where $A=\operatorname{Pic}(\mathrm{C})$ and $N=\operatorname{Aut}(S)$. Finally, use Proposition 7.12. This shows the existence of an $A$-trivialization $\sigma$ of $\mathcal{C}$ with respect to $X$.

Suppose now that $\sigma^{\prime}$ is another $A$-trivialization of $\mathcal{C}$ with respect to $X$. Define

$$
\theta_{a, b}=D\left(\left(\sigma_{a, b}^{\prime}\right)^{-1} \circ \sigma_{a, b}\right) \in \operatorname{Aut}(S)
$$

for each $a, b \in A$. Note the resulting formula $\sigma_{a, b}^{\prime}=\sigma_{a, b} \hat{\otimes} \theta_{a, b}$. Condition (1) in the definition of $A$-trivialization shows that $\theta_{a, b}=\mathrm{id}_{S}$ if either $a$ or $b$ is zero. Take the pentagonal diagram in condition (2) for $\sigma$ and let $C$ denote a composition going around the pentagon; let $C^{\prime}$ denote the corresponding composition for $\sigma^{\prime}$. Note that $C=C^{\prime}=$ id by commutativity of these diagrams. But if we replace each $\sigma_{a, b}^{\prime}$ appearing in $C^{\prime}$ with $\sigma_{a, b} \hat{\otimes} \theta_{a, b}$, then all of the $\theta$ 's can be moved outside the composition by Remark 4.3. This shows that $C^{\prime}=C \hat{\otimes}(\delta \theta)(a, b, c)$. Since $C=C^{\prime}=$ id we get that $\delta \theta(a, b, c)=\operatorname{id}_{S}$ for every $a, b, c \in A$. So $\theta \in Z^{2}(A ; \operatorname{Aut}(S))_{\text {norm }}$, and moreover it is easy to see that this gives a bijection between $A$-trivializations and elements of $Z^{2}(A ; \operatorname{Aut}(S))_{\text {norm }}$.

Finally, we have seen how the trivializations $\sigma$ and $\sigma^{\prime}$ each give rise to a ring structure on $\pi_{*}^{A}(S)$; write these as $\pi_{*}^{A}(S)_{\sigma}$ and $\pi_{*}^{A}(S)_{\sigma^{\prime}}$. Recall from the beginning of this section that a standard isomorphism between these rings depends on a fixed map of sets $u: A \rightarrow \operatorname{Aut}(S)$. Let $F_{u}: \pi_{*}^{A}(S) \rightarrow \pi_{*}^{A}(S)$ be the map of $A$-graded abelian groups which sends $f: X_{a} \rightarrow S$ to $f \cdot u(a)$. It is routine to check that $F_{u}$ gives a ring isomorphism $\pi_{*}^{A}(S)_{\sigma} \rightarrow \pi_{*}^{A}(S)_{\sigma^{\prime}}$ if and only if $\delta u=\theta$, therefore completing the proof. 


\section{Appendix: A short motivic application}

Here we give the proof of Proposition 1.19. We concentrate on the basic idea, ignoring technical details about the foundations.

Let $\operatorname{Ho}(S p)$ and $\operatorname{Ho}(\mathcal{M}$ otSp) denote the stable homotopy category and the motivic stable homotopy category over $\mathbb{C}$, respectively. These both have symmetric monoidal structures, with the units written $S^{0}$ and $S^{0,0}$, respectively. There is a realization functor $\psi: \operatorname{Ho}(\mathcal{M}$ otSp $) \rightarrow \operatorname{Ho}(S p)$ that is strong monoidal. Let $X_{1}=S^{1,0}$ and $X_{2}=S^{1,1}$ be the standard motivic spheres, and write $S^{1}$ for the classical suspension spectrum of the circle. Choose a model for $S^{-1}$ in $\operatorname{Ho}(S p)$ and an isomorphism $\eta: S^{0} \rightarrow S^{-1} \wedge S^{1}$. Choose inverses $X_{1}^{*}$ and $X_{2}^{*}$, and let us assume for simplicity that $\psi\left(X_{1}^{*}\right)=S^{-1}$ and $\psi\left(X_{2}^{*}\right)=S^{-1}$ (equalities instead of merely isomorphisms). A little thought shows that one can choose isomorphisms $\eta_{1}: S^{0,0} \rightarrow X_{1}^{*} \wedge X_{1}$ and $\eta_{2}: S^{0,0} \rightarrow X_{2}^{*} \wedge X_{2}$ that map to $\eta$ under $\psi$.

Below we will write $Z=S^{1}$ to avoid having to write double exponents like $\left(S^{1}\right)^{a}$.

Proof of Proposition 1.19 Let $f: X_{1}^{a} \wedge X_{2}^{b} \rightarrow S$ and $g: X_{1}^{r} \wedge X_{2}^{s} \rightarrow S$. Then $f \cdot g$ is the composite

$$
X_{1}^{a+r} \wedge X_{2}^{b+s} \stackrel{\phi}{\longrightarrow} X_{1}^{a} \wedge X_{2}^{b} \wedge X_{1}^{r} \wedge X_{2}^{s} \stackrel{f \wedge g}{\longrightarrow} S \wedge S=S .
$$

The canonical isomorphism $\phi$ commutes the $X_{2}^{b}$ past the $X_{1}^{r}$ and then simplifies the resulting monomial by using associativity and the $\eta$ and $\hat{\eta}$ maps (but without any more commutations). If we apply $\psi$ to this composite then we get the analogous composite

$$
Z^{a+r+b+s} \longrightarrow Z^{a} \wedge Z^{b} \wedge Z^{r} \wedge Z^{s} \stackrel{\psi(f) \wedge \psi(g)}{\longrightarrow} S \wedge S=S .
$$

Note that the first map in the composite is not a canonical map anymore, and so we have dropped the label $\phi$. Rather, this map commutes the $Z^{r}$ past the $Z^{b}$. If we were to compute $\psi(f) \cdot \psi(g)$ in $\pi_{*}(S)$, however, we would get the composite

$$
Z^{a+r+b+s} \stackrel{\phi}{\longrightarrow} Z^{a} \wedge Z^{b} \wedge Z^{r} \wedge Z^{s} \stackrel{\psi(f) \wedge \psi(g)}{\longrightarrow} S \wedge S=S .
$$

So we obtain a commutative diagram

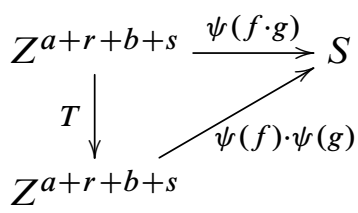


where $T$ is the composite

$$
Z^{a+r+b+s} \stackrel{\phi}{\longrightarrow} Z^{a} \wedge Z^{r} \wedge Z^{b} \wedge Z^{s} \stackrel{1 \wedge t_{r, b} \wedge 1}{\longrightarrow} Z^{a} \wedge Z^{b} \wedge Z^{r} \wedge Z^{s} \stackrel{\phi}{\longrightarrow} Z^{a+r+b+s} .
$$

Using Lemma 6.10 the triangle gives $\psi(f \cdot g)=\psi(f) \cdot \psi(g) \cdot D(T)$. We next compute that

$$
D(T)=[T]_{r}=[\phi]_{r} \circ\left[1 \wedge t_{r, b} \wedge 1\right]_{r} \circ[\phi]_{r}=\mathrm{id} \circ\left[t_{r, b}\right]_{r} \circ \mathrm{id}=\tau_{r, b}=\tau_{1}^{r b}=(-1)^{r b}
$$

(the first, second and third equalities are by Proposition 6.5, and the fifth equality is by Proposition 6.6). This yields the desired result; one only needs to remember that the motivic bigrading is set up so that $f \in \pi_{a+b, b}(S)$ and $g \in \pi_{r+s, s}(S)$, and then one recovers the formula from the statement of the proposition.

A.1 Remark Note that in the setup of motivic homotopy groups we chose $X_{1}=S^{1,0}$ and $X_{2}=S^{1,1}$. The sign in Proposition 1.19 actually depends on this choice. We leave it as an exercise to check that if we had chosen $X_{1}=S^{1,1}$ and $X_{2}=S^{1,0}$ then the sign rule would be $\psi(f g)=(-1)^{(a-b) s} \psi(f) \psi(g)$ for $f \in \pi_{a, b}(S)$ and $g \in \pi_{r, s}(S)$. This shows how sensitive sign formulas are to the choices in the bookkeeping.

\section{References}

[1] J F Adams, Prerequisites (on equivariant stable homotopy) for Carlsson's lecture, from: "Algebraic topology", (I Madsen, B Oliver, editors), Lecture Notes in Math. 1051, Springer, Berlin (1984) 483-532 MR764596

[2] A M Cegarra, E Khmaladze, Homotopy classification of graded Picard categories, Adv. Math. 213 (2007) 644-686 MR2332605

[3] P Deligne, La formule de dualité globale, from: "Sém. Geom. algébrique Bois-Marie 1963/64, SGA 4", Lect. Notes Math. 3, Springer-Verlag (1973) 481-587

[4] S Eilenberg, S Mac Lane, Cohomology theory of Abelian groups and homotopy theory, II, Proc. Nat. Acad. Sci. U. S. A. 36 (1950) 657-663 MR0039252

[5] S Eilenberg, S Mac Lane, On the groups $H(\Pi, n)$, II, Ann. of Math. 60 (1954) 49-139 MR0065162

[6] A Fröhlich, C T C Wall, Graded monoidal categories, Compositio Math. 28 (1974) 229-285 MR0349804

[7] X S Hoàng, Gr-categories, PhD thesis, Université Paris VII (1975)

[8] N Johnson, A M Osorno, Modeling stable one-types, Theory Appl. Categ. 26 (2012) 520-537 MR2981952

[9] A Joyal, R Street, Braided tensor categories, Adv. Math. 102 (1993) 20-78 MR1250465 
[10] A Joyal, R Street, D Verity, Traced monoidal categories, Math. Proc. Cambridge Philos. Soc. 119 (1996) 447-468 MR1357057

[11] G M Kelly, M L Laplaza, Coherence for compact closed categories, J. Pure Appl. Algebra 19 (1980) 193-213 MR593254

[12] L G Lewis, Jr, M A Mandell, Equivariant universal coefficient and Künneth spectral sequences, Proc. London Math. Soc. 92 (2006) 505-544 MR2205726

[13] S Mac Lane, Categories for the working mathematician, 2nd edition, Graduate Texts in Mathematics 5, Springer, New York (1998) MR1712872

[14] JP May, Equivariant homotopy and cohomology theory, CBMS Regional Conference Series in Mathematics 91, published for the CBMS, Washington, DC (1996) MR1413302

[15] J P May, J Sigurdsson, Parametrized homotopy theory, Mathematical Surveys and Monographs 132, Amer. Math. Soc. (2006) MR2271789

[16] K Ponto, M Shulman, Traces in symmetric monoidal categories arXiv:1107.6032

[17] V Voevodsky, $\mathbf{A}^{1}$-homotopy theory, from: "Proceedings of the International Congress of Mathematicians, Vol. I", (S D Chatterji, editor), Extra Vol. I (1998) 579-604 MR1648048

Department of Mathematics, University of Oregon

Fenton Hall, Eugene, OR 97403, USA

ddugger@math . uoregon . edu

http://math.uoregon.edu/ ddugger

Received: 5 March 2013 Revised: 8 October 2013 